\title{
Factors affecting microbial lipids production by Yarrowia lipolytica strains from volatile fatty acids: Effect of co-substrates, operation mode and oxygen
}

\author{
Ana S. Pereira, Sílvia M. Miranda, Marlene Lopes, Isabel Belo* \\ CEB-Centre of Biological Engineering, University of Minho, Campus de Gualtar, 4710-057 Braga, Portugal
}

\section{A R T I C L E I N F O}

\section{Keywords:}

Yarrowia lipolytica

Volatile fatty acids

Acetate

Propionate

Butyrate

Microbial lipids

\begin{abstract}
A B S T R A C T
Volatile fatty acids (VFAs), which can be generated by acidogenesis of organic wastes, are important building blocks for chemicals production, and are intermediates in many bioprocesses such as microbial lipids production. Important factors affecting the bioconversion of VFAs (acetate, propionate and butyrate) by Yarrowia lipolytica W29 and NCYC 2904 for growth and lipids accumulation were studied. Yarrowia lipolytica grew efficiently in VFAs-based media, but lipids production was enhanced by the addition of co-substrates (glucose or glycerol) in batch cultures. A two-stage batch culture - growth phase on glucose, followed by VFAs addition, improved lipids accumulation. Lipids concentrations of $2.3 \mathrm{~g} \cdot \mathrm{L}^{-1}$ and $3.5 \mathrm{~g} \cdot \mathrm{L}^{-1}$ were obtained with this mode of operation, with addition of $18 \mathrm{~g} \cdot \mathrm{L}^{-1}$ VFAs, for Y. lipolytica W29 and NCYC 2904, respectively. For the first time, it was demonstrated that oxygen mass transfer is a crucial factor for lipids production by $Y$. lipolytica from VFAs. Intracellular lipids produced by Y. lipolytica strains were mainly composed by oleic and linoleic acids, similar to common vegetable oils, making these lipids suitable for biodiesel production. Moreover, margaric acid, which may improve biodiesel properties, was only detected in propionate medium. The strategies studied herein will contribute to the feasibility of using VFAs as low-cost feedstock for microbial lipids production by $Y$. lipolytica strains.
\end{abstract}

\section{Introduction}

The growth of worldwide population and consequent human activity lead to a continuous increase in residues generation. In 2018, 220 million tons of municipal wastes (about $492 \mathrm{~kg}$ per capita) were generated in the European Union (PORTDATA, 2020a) and only $47 \%$ was recycled (PORTDATA, 2020b). In some countries, more than $70 \%$ of municipal wastes were incinerated or disposed of in landfill with negative environmental impacts, including water and soil pollution due to the leachate and greenhouse gases emission resulting from decomposition of biodegradable waste (Scherhaufer et al., 2018). Anaerobic digestion (AD) is an efficient technology for the treatment of organic fraction of municipal wastes with great potential to produce biofuels (biogas, biohydrogen) and chemicals, such as volatile fatty acids (Gameiro et al., 2016; Strazzera et al., 2018). Volatile fatty acids (VFAs), produced in acidogenesis and acetogenesis steps of $\mathrm{AD}$ of organic wastes, are considered important building blocks for chemical industries, since they are precursors of reduced compounds (alcohols, aldehydes and ketones). Moreover, VFAs can be used as substrate for several microbiological processes, as carbon and energy sources for the production of added-value compounds (Lee et al., 2014; Strazzera et al., 2018).

The non-conventional yeast Yarrowia lipolytica has the ability to grow and accumulate lipids up to $50 \%$ of its cell dry mass from wastes and by-products of agro-industrial sector (Lopes et al., 2018; Papanikolaou et al., 2007; Tsigie et al., 2012). Some studies on lipids production by $Y$. lipolytica have been performed with VFAs as sole carbon source, and most of them reported that high VFAs concentrations (above $5 \mathrm{~g} \cdot \mathrm{L}^{-1}$ ) had an inhibitory effect on yeast growth (Fontanille et al., 2012; Gao et al., 2017; Kolouchová et al., 2015; Rodrigues and Pais, 2000). Lipids accumulated by Y. lipolytica using VFAs as carbon source have similar fatty acid composition to common vegetable oils, making them a suitable raw material for biodiesel production. Conventionally, biodiesel is produced by transesterification of lipids extracted from a variety of feedstocks, including plant/vegetable oils, but the high cost and limited supply of these raw materials are the major hindrance for

\footnotetext{
* Corresponding author.

E-mail address: ibelo@deb.uminho.pt (I. Belo).
} 
commercial production of biodiesel. The cost of these feedstocks corresponds to $75 \%$ of total cost of biodiesel produced from edible and non-edible vegetable oils (Gaeta-Bernardi and Parente, 2016). Furthermore, pure vegetable oils are widely employed for food purposes, reducing their availability for biodiesel production. Therefore, an alternative approach for economical and sustainable biodiesel production may be based on the use of microbial lipids produced from organic waste-derived VFAs by oleaginous microorganisms, favoring the circular economy. Thus, this work addresses the factors that influence the growth and microbial lipids production from VFAs by $Y$. lipolytica strains, comparing two strains performance (W29 and NCYC 2904). Batch cultures in VFAs and with glucose or glycerol as co-substrate were performed, as well as two-stage batch cultures (first stage - growth in glucose; second stage - lipids production in VFAs) in order to improve yeast growth and lipids synthesis. $Y$. lipolytica is a strictly aerobic yeast and the culture oxygenation is an important parameter affecting yeast metabolism. Besides cellular growth (Lopes et al., 2009), the production of lipase (Lopes et al., 2019), critic acid (Ferreira et al., 2016) and $\gamma$-decalactone (Braga et al., 2015) by Y. lipolytica W29 were improved by the increase of oxygen transfer rate from air to culture medium. The intracellular accumulation of lipids from lard (Lopes et al., 2018) and waste cooking oils (Lopes et al., 2019) were also affected by dissolved oxygen concentration. Thereby, the effect of oxygen availability on microbial lipids production from VFAs was assessed, for the first time, in a laboratory-scale bioreactor (stirred tank bioreactor).

\section{Material and methods}

\subsection{Yeast strains}

Yarrowia lipolytica W29 (ATCC 20460) and Y. lipolytica NCYC 2904 cells, previously grown in YPD medium $\left(20 \mathrm{~g} \cdot \mathrm{L}^{-1}\right.$ peptone, $10 \mathrm{~g} \cdot \mathrm{L}^{-1}$ yeast extract and $20 \mathrm{~g} \cdot \mathrm{L}^{-1}$ glucose), were maintained in cryo-stocks ( $800 \mu \mathrm{L}$ of yeast culture and $200 \mu \mathrm{L}$ of glycerol $99.5 \%$ ) and stored at $80{ }^{\circ} \mathrm{C}$ until used to prepare inoculum cultures.

\subsection{Batch cultures}

Batch cultures were carried out in $500 \mathrm{~mL}$ baffled Erlenmeyer flasks containing $200 \mathrm{~mL}$ of VFA-based medium, composed by $5 \mathrm{~g} \cdot \mathrm{L}^{-1} \mathrm{VFA}$ (acetate, propionate or butyrate), $0.5 \mathrm{~g} \cdot \mathrm{L}^{-1}$ yeast extract, $1.7 \mathrm{~g} \cdot \mathrm{L}^{-1} \mathrm{YNB}$ (without amino acids and without ammonium sulfate) and ammonium sulfate in variable amounts according to carbon source in order to obtain an initial $\mathrm{C} / \mathrm{N}$ ratio of 75. Initial medium $\mathrm{pH}$ was adjusted to 6 and batch cultures were carried out at $27^{\circ} \mathrm{C}$ and $170 \mathrm{rpm}$ in an orbital incubator. Each Erlenmeyer flask was inoculated with a pre-culture grown overnight in YPD medium. Yeast cells were harvested by centrifugation and resuspended (at an initial cell density of $0.5 \mathrm{~g} \cdot \mathrm{L}^{-1}$ ) in culture medium.

Additionally, batch cultures with addition of $5 \mathrm{~g} \cdot \mathrm{L}^{-1}$ glucose to each VFA-based medium were also carried out to study the effect of the cosubstrate on $Y$. lipolytica W29 growth and lipids accumulation. Since the fermentative broth obtained in acidogenesis of organic wastes is composed by various VFAs, the ability of $Y$. lipolytica to assimilate a mixture of VFAs was also evaluated. Thus, batch experiments were performed with Y. lipolytica W29 and NCYC 2904 strain growing in a VFAs-based medium composed by mixture of VFAs $\left(2 \mathrm{~g} \cdot \mathrm{L}^{-1}\right.$ acetate, $2 \mathrm{~g} \cdot \mathrm{L}^{-1}$ propionate and $2 \mathrm{~g} \cdot \mathrm{L}^{-1}$ butyrate), $20 \mathrm{~g} \cdot \mathrm{L}^{-1}$ co-substrate (glucose or glycerol), $0.5 \mathrm{~g} \cdot \mathrm{L}^{-1}$ yeast extract, $1.7 \mathrm{~g} \cdot \mathrm{L}^{-1} \mathrm{YNB}$ and ammonium sulfate (the amount varied depending on co-substrate type, to obtain a $\mathrm{C} / \mathrm{N}$ ratio of 75 ).

\subsection{Two-stage batch cultures}

Two-stage batch cultures were performed in $500 \mathrm{~mL}$ baffled Erlenmeyer flasks filled with $200 \mathrm{~mL}$ of medium. In two-stage batch cultures, the accumulation of intracellular lipids by Y. lipolytica W29 cells from
VFAs was preceded by a growth phase without VFAs. This first stage occurred in glucose-based medium composed by: $20 \mathrm{~g} \cdot \mathrm{L}^{-1}$ glucose, $0.5 \mathrm{~g} \cdot \mathrm{L}^{-1}$ yeast extract, $1.7 \mathrm{~g} \cdot \mathrm{L}^{-1} \mathrm{YNB}$ and $0.503 \mathrm{~g} \cdot \mathrm{L}^{-1}$ ammonium sulfate for biomass production. After $24 \mathrm{~h}$ of cell growth, corresponding to the final of exponential growth phase, a mixture of acetate, propionate and butyrate, with equal concentration of each one, was added to the medium (second stage). In this strategy, several total VFAs concentration $\left(6 \mathrm{~g} \cdot \mathrm{L}^{-1}, 18 \mathrm{~g} \cdot \mathrm{L}^{-1}, 24 \mathrm{~g} \cdot \mathrm{L}^{-1}\right.$ and $\left.30 \mathrm{~g} \cdot \mathrm{L}^{-1}\right)$ were tested. The twostage batch culture using a mixture with $18 \mathrm{~g} \cdot \mathrm{L}^{-1}$ of VFAs was also performed with Y. lipolytica NCYC 2904.

\subsection{Bioreactor batch experiments}

To evaluate the effect of oxygen volumetric mass transfer coefficient $\left(k_{\mathrm{L}} a\right)$ on cell growth and lipids accumulation by $Y$. lipolytica W29 and $Y$. lipolytica NCYC 2904, several batch cultures were carried out in a 5-L stirred tank bioreactor (BIOSTAT ${ }^{\circledR}$ B Plus, Sartorius) at $27^{\circ} \mathrm{C}$, varying the specific air flow rate $(0.5 \mathrm{vvm}-1.5 \mathrm{vvm})$ and agitation rate (200 rpm - $600 \mathrm{rpm}$ ). Yeast cells, pre-grown overnight in YPD medium, were centrifuged and resuspended (at an initial cell density of $0.5 \mathrm{~g} \cdot \mathrm{L}^{-1}$ ) in $2.5 \mathrm{~L}$ of VFA-based medium consisted of: VFAs mixture $\left(2 \mathrm{~g} \cdot \mathrm{L}^{-1}\right.$ of each VFA), $20 \mathrm{~g} \cdot \mathrm{L}^{-1}$ glucose, $0.5 \mathrm{~g} \cdot \mathrm{L}^{-1}$ yeast extract, $1.7 \mathrm{~g} \cdot \mathrm{L}^{-1} \mathrm{YNB}$ and $0.685 \mathrm{~g} \cdot \mathrm{L}^{-1}$ ammonium sulfate. $\mathrm{pH}$ of culture medium was maintained at 6 by the addition of hydrochloric acid $2 \mathrm{M}$ or sodium hydroxide $2 \mathrm{M}$. Dissolved oxygen concentration was measured with an optical probe (InPro 6860i, Mettler Toledo, USA) and the respective meter (Solaris Biotechnology SRL, Italy).

\section{5. $k_{L}$ a measurement}

To calculate the experimental $k_{\mathrm{L}} a$ at different aeration conditions used in yeast cultures, a static gassing-out technique was employed in blank assays (culture medium without cells). This method consists of following the dissolved oxygen concentration in liquid until saturation at specific conditions of air flow and agitation rates, after a preliminary gassing-out with nitrogen to remove the oxygen in medium (Ferreira et al., 2016).

\subsection{Analytical methods}

At appropriate intervals, culture samples were taken for the measurement of $\mathrm{pH}$, and the quantification of cell mass, glucose, glycerol and VFAs concentration. At the end of experiments, cellular lipids content and long chain fatty acids were determined and monitored by microscopic visualization.

Cell mass concentration was quantified by optical density at $600 \mathrm{~nm}$ and converted to cell dry weight $\left(\mathrm{g} \cdot \mathrm{L}^{-1}\right)$ by a calibration curve. Glucose, glycerol and VFAs (acetate, propionate and butyrate) concentration were measured by high-performance liquid chromatography (HPLC) using an Aminex HPX-87H column $(300 \mathrm{~mm} \times 7.8 \mathrm{~mm}, 8 \mu \mathrm{m}$ particle size) at $60{ }^{\circ} \mathrm{C}$ and coupled with refractive index and ultra-violet detectors. The mobile phase was sulfuric acid $5 \mathrm{mM}$ at $0.5 \mathrm{~mL} \cdot \mathrm{min}^{-1}$ flow rate.

Microbial lipids were extracted from lyophilized cells using a mixture of methanol and chloroform and quantified by phospho-vanillin colorimetric method (Lopes et al., 2018). Briefly, ten milligrams of lyophilized cells were suspended in $2 \mathrm{~mL}$ of methanol and chloroform $(1: 1, v / v)$, vortexed for $3 \mathrm{~min}$ and $250 \mu \mathrm{L}$ of supernatant was collected and heated at $100{ }^{\circ} \mathrm{C}$ until the total evaporation of solvents. Then, $100 \mu \mathrm{L}$ of sulfuric acid $98 \%$ was added and the solution was incubated for $15 \mathrm{~min}$ at $100{ }^{\circ} \mathrm{C}$. After cooling to room temperature, $2.4 \mathrm{~mL}$ of phospho-vanillin reagent (vanillin dissolved in orthophosphoric acid 85 $\%$ ) was added and the mixture rested for $15 \mathrm{~min}$. The phospho-vanillin solution reacted with lipids producing a pink color, whose intensity was quantified by measuring the absorbance at $490 \mathrm{~nm}$. This absorbance was converted to lipids concentration $\left(\mathrm{g} \cdot \mathrm{L}^{-1}\right)$ by a calibration curve, using 

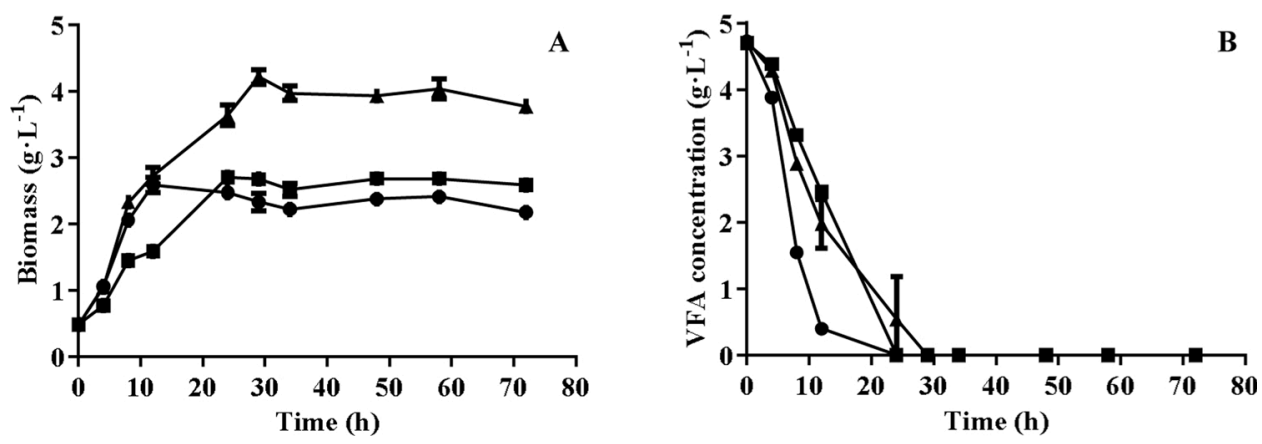

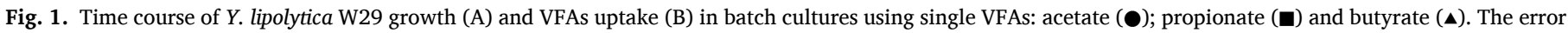
bars represent the standard deviation of two independent replicates.

olive oil as standard.

The fatty acid composition of microbial lipids was analyzed by gas chromatography (GC). Prior to GC analysis, fatty acids were extracted from lyophilized cells with chloroform and transformed into their methyl esters (FAMEs) with a mixture of methanol acidified with sulfuric acid (85:15, v/v), using pentadecanoic acid (C15:0) as internal standard (Lopes et al., 2019). The organic phase containing FAMEs was analyzed in a chromatograph (CP-3800 gas chromatograph, Varian Inc., USA) fitted with FID detector and TRACSIL TR-WAX capillary column ( $30 \mathrm{~m} \times 0.25 \mathrm{~mm} \times 0.25 \mathrm{~mm}$, Teknokroma, Spain). The temperatures of injector and detector were, respectively, $220^{\circ} \mathrm{C}$ and $250{ }^{\circ} \mathrm{C}$, and helium at $1 \mathrm{~mL} \cdot \mathrm{min}^{-1}$ was used as carrier gas. For the complete separation of FAMEs, a temperature gradient was used: $50{ }^{\circ} \mathrm{C}$ for $2 \mathrm{~min}$, followed by an increase of $10^{\circ} \mathrm{C} \cdot \mathrm{min}^{-1}$ up to $225^{\circ} \mathrm{C}$, which was maintained by $10 \mathrm{~min}$. The identification of FAMEs was carried out by comparison of retention times with a mixture of FAMEs standards. The relative amount of each fatty acid $(\% \mathrm{w} / \mathrm{w})$ was defined as the ratio between its concentration $\left(\mathrm{g} \cdot \mathrm{L}^{-1}\right)$ and the total fatty acids detected in the sample.

Lipids accumulated intracellularly in lipid bodies were visualized by fluorescence microscopy after cells staining with Nile red. Sampled cellular suspensions were centrifuged, washed twice with PBS $0.1 \mathrm{M}$ and resuspended in PBS $50 \mathrm{mM}$ to a final absorbance of $5(\lambda=600 \mathrm{~nm})$ and then stained with a Nile red solution. The staining solution, containing $0.1 \mathrm{~g} \cdot \mathrm{L}^{-1}$ Nile red in acetone, was added to the cellular suspension in the proportion of 1:10 $(\mathrm{v} / \mathrm{v})$ and incubated at room temperature for $1 \mathrm{~h}$. Stained cells were then visualized in an Olympus BX 51 microscope using an exciting wavelength of $470 \mathrm{~nm}-490 \mathrm{~nm}$.

\subsection{Kinetic parameters}

The biomass yield $\left(Y_{\mathrm{X} / \mathrm{S}}, \mathrm{g} \cdot \mathrm{g}^{-1}\right)$ was calculated according to Eq. 1, where $X_{\mathrm{f}}$ is the final biomass concentration, $X_{\mathrm{i}}$ is the initial biomass concentration, $S_{\mathrm{i}}$ is the initial substrate concentration and $S_{\mathrm{f}}$ is the substrate concentration at the end of growth.
$Y_{\mathrm{X} / \mathrm{S}}=\frac{X_{f}-X_{i}}{S_{i}-S_{f}}$

The lipids yield $\left(Y_{\mathrm{L} / \mathrm{S}}, \mathrm{g} \cdot \mathrm{g}^{-1}\right)$ was calculated according to Eq. 2, where $L_{\mathrm{f}}$ is the concentration of lipids at time t $(\mathrm{h}), L_{\mathrm{i}}$ is the initial lipids concentration, $S_{\mathrm{i}}$ is the initial substrate concentration and $S_{\mathrm{f}}$ is the substrate concentration at time (h).

$Y_{\mathrm{L} / \mathrm{s}}=\frac{L_{f}-L_{i}}{S_{i}-S_{f}}$

The VFAs uptake rate $\left(R_{\text {VFAs }}, g \cdot \mathrm{L}^{-1} \cdot \mathrm{h}^{-1}\right)$ corresponds to the slope of VFAs concentration through the time in the linear zone.

\subsection{Reproducibility of results}

All experiments were carried out in duplicate and the results represent the mean of two independent biological replicates. Statistical analysis was performed using Tukey's test (Statgraphics Centurion XVI software, Version 16.2.04).

\section{Results and discussion}

\subsection{Batch cultures of Y. lipolytica on VFAs-based medium}

The ability of $Y$. lipolytica W29 to use VFAs as sole carbon and energy sources to grow and accumulate lipids was firstly evaluated in Erlenmeyer flask experiments. Acetate, propionate and butyrate, which are usually the most common VFAs obtained in acidogenesis step of organic wastes anaerobic digestion (Gameiro et al., 2016), were used in batch cultures. Yarrowia lipolytica W29 was able to grow in each VFA, although higher final biomass was attained in butyrate-based medium and no significant differences were observed in yeast growth with acetate and propionate (Fig. 1A). In fact, the biomass yield (cell mass per VFA mass consumed) obtained with butyrate was 1.8- and 1.6-fold higher than the values attained with acetate and propionate, respectively (Table 1).

Table 1

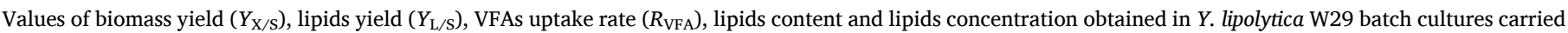

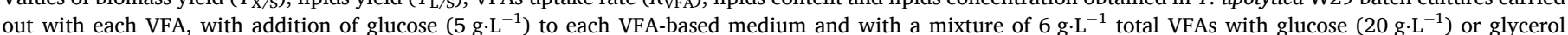

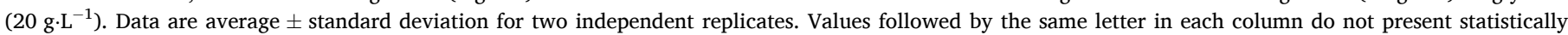
significant differences (95\% confidence level).

\begin{tabular}{|c|c|c|c|c|c|}
\hline Carbon source & $Y_{\mathrm{X} / \mathrm{S}}\left(\mathrm{g} \cdot \mathrm{g}^{-1}\right)$ & $Y_{\mathrm{L} / \mathrm{S}}\left(\mathrm{g} \cdot \mathrm{g}^{-1}\right)$ & $R_{\mathrm{VFA}}\left(\mathrm{g} \cdot \mathrm{L}^{-1} \cdot \mathrm{h}^{-1}\right)$ & Lipids content $(\%, \mathrm{w} / \mathrm{w})$ & Lipids concentration $\left(\mathrm{g} \cdot \mathrm{L}^{-1}\right)$ \\
\hline Acetate & $0.40 \pm 0.01^{\mathrm{a}}$ & $0.037 \pm 0.001^{\mathrm{ab}}$ & $0.38 \pm 0.02^{\mathrm{a}}$ & $7.3 \pm 0.4^{\mathrm{a}}$ & $0.17 \pm 0.01^{\mathrm{a}}$ \\
\hline Propionate & $0.46 \pm 0.01^{\mathrm{ab}}$ & $0.045 \pm 0.002^{\mathrm{ab}}$ & $0.20 \pm 0.00^{b}$ & $7.9 \pm 0.6^{\mathrm{ab}}$ & $0.21 \pm 0.01^{\mathrm{a}}$ \\
\hline Butyrate & $0.729 \pm 0.002^{c}$ & $0.096 \pm 0.005^{c}$ & $0.164 \pm 0.001^{c}$ & $11.5 \pm 0.5^{\mathrm{cd}}$ & $0.45 \pm 0.02^{b}$ \\
\hline Acetate + Glucose & $0.54 \pm 0.01^{b d}$ & $0.050 \pm 0.003^{b d}$ & $0.34 \pm 0.00^{\mathrm{a}}$ & $9.1 \pm 1.1$ be & $0.46 \pm 0.03^{b}$ \\
\hline Propionate + Glucose & $0.39 \pm 0.03^{a}$ & $0.045 \pm 0.008^{a b}$ & $0.132 \pm 0.003^{d}$ & $9.6 \pm 1.3^{\mathrm{e}}$ & $0.41 \pm 0.07^{\mathrm{b}}$ \\
\hline Butyrate + Glucose & $0.72 \pm 0.01^{c}$ & $0.090 \pm 0.001^{c}$ & $0.202 \pm 0.005^{b}$ & $12.4 \pm 1.2^{\mathrm{d}}$ & $0.85 \pm 0.01^{c}$ \\
\hline VFAs + Glucose & $0.63 \pm 0.07^{\mathrm{cd}}$ & $0.06 \pm 0.02^{d}$ & $0.21 \pm 0.01^{b}$ & $10 \pm 2$ bce & $1.42 \pm 0.31^{\mathrm{d}}$ \\
\hline VFAs + Glycerol & $0.41 \pm 0.03^{\mathrm{a}}$ & $0.033 \pm 0.002^{\mathrm{a}}$ & $0.16 \pm 0.01^{c}$ & $7.9 \pm 0.4^{\text {abe }}$ & $0.82 \pm 0.02^{c}$ \\
\hline
\end{tabular}



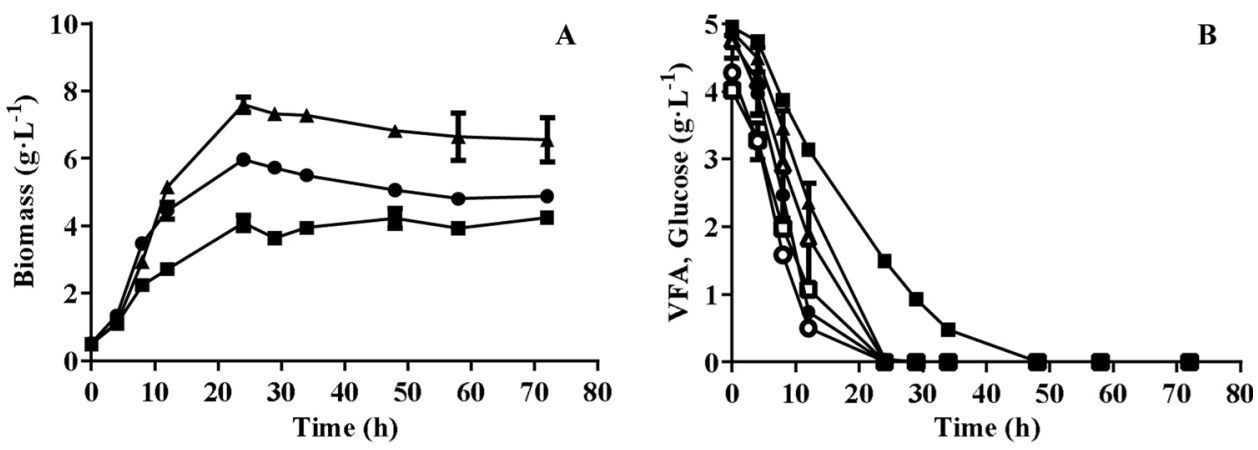

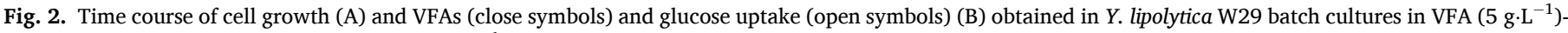

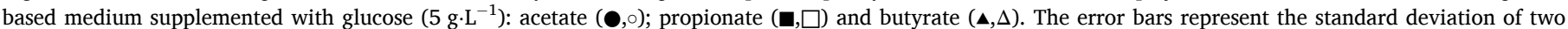
independent replicates.

Apparently, increasing VFA chain length led to the rise of biomass production. Llamas et al. (2020) also observed that the final cellular concentration of $Y$. lipolytica CECT1240, growing in several VFAs (C2 to C6), increased proportionally with chain length. The maximum biomass obtained in caproate (C6), for example, was approximately 2 -fold of that attained in acetate (C2). Moreover, a 3-fold improvement in biomass yield was observed when the authors used butyrate as carbon source, instead of acetate. Studies with other species also concluded that the use of butyrate as carbon source for Cryptoccocus curvatus gives rise to higher biomass yield comparatively to acetate and propionate (Liu et al., 2017; Park et al., 2017; Zheng et al., 2012). By contrast, some works showed that higher cellular growth was obtained in acetate comparatively to other VFAs (Gao et al., 2017, 2020; Kolouchová et al., 2015).

In all experiments, no lag phase of cellular growth was observed, and the stationary phase was reached approximately after $27 \mathrm{~h}$ of yeast cultivation, which corresponds to the time of complete depletion of each VFA from culture medium (Fig. 1B). The consumption profiles show that VFA consumption is always associated with yeast growth and suggest that acetate was consumed faster than other VFAs. The consumption rates calculated for each VFA confirm that there were statistical differences between uptake rates of VFAs, and acetate was consumed $47 \%$ faster than other VFAs (Table 1). It is worth to notice that uptake rates reported herein are considerable higher than others found in the literature. In Y. lipolytica CICC 31596 cultures, $2.5 \mathrm{~g} \cdot \mathrm{L}^{-1}$ of acetate, propionate and butyrate were totally consumed only after $48 \mathrm{~h}, 84 \mathrm{~h}$ and $96 \mathrm{~h}$, respectively (Gao et al., 2017). Differences in utilization rates of VFAs may be attributed to their different metabolic routes inside $Y$. lipolytica cells. While acetate is converted directly into acetyl-CoA by acetyl-CoA synthase propionate is firstly converted to propionyl-CoA that enters in the methyl citrate cycle to produce pyruvate, which is then decarboxylated to acetyl-CoA. Butyrate is converted to butyryl-CoA and then to acetyl-CoA via $\beta$-oxidation. The acetyl-CoA produced from all VFAs can be directly incorporated into lipids during de novo synthesis or transported into the mitochondria and enter in the TCA cycle for energy production. Acetyl-CoA units can also incorporate the glyoxylate shunt and gluconeogenesis pathway (Liu et al., 2016; Vajpeyi and Chandran, 2015).

The highest lipid production was reached in butyrate-based medium (Table 1), that was also reported for other yeast species, in which butyrate in relatively low concentrations was more suitable for lipids synthesis by C. curvatus than acetate and propionate (Liu et al., 2017; Park et al., 2017). In high alkaline conditions (pH 10), butyrate was also the best substrate for lipids production by Y. lipolytica CICC 31596 (Gao et al., 2020). By contrast, in slightly acidic conditions (pH 6), a $13 \%$ and $38 \%$ enhancement on lipids synthesis was attained in acetate comparatively with propionate and butyrate, respectively (Gao et al., 2017).

In general, oleaginous yeasts have the ability to accumulate more than $20 \%$ of their cell dry weight as intracellular lipids. The content, concentration and yield of lipids on Table 1 were low comparatively to others reported in the literature for microbial lipids production by Y. lipolytica strains from VFAs (Liu et al., 2017; Gao et al., 2017, 2020), glucose (Katre et al., 2012), animal fat (Lopes et al., 2018), waste cooking oils (Lopes et al., 2019) or glycerol (Poli et al., 2014; Sara et al., 2016). In these substrates, lipids content ranges from $20 \%(\mathrm{w} / \mathrm{w})$ to 60 $\%(\mathrm{w} / \mathrm{w})$, lipids concentration varies from $0.6 \mathrm{~g} \cdot \mathrm{L}^{-1}$ to $14 \mathrm{~g} \cdot \mathrm{L}^{-1}$ and lipids yield ranges from $0.1 \mathrm{~g} \cdot \mathrm{g}^{-1}$ to $0.2 \mathrm{~g} \cdot \mathrm{g}^{-1}$. Though VFAs have higher theoretical conversion efficiencies for lipid synthesis than other substrates with shorter metabolic pathways (Gong et al., 2016; Zheng et al., 2012), many nutritional and environmental factors, such as VFAs concentration, $\mathrm{pH}$ and yeast strain, can strongly affect lipids accumulation (Gao et al., 2017, 2020; Katre et al., 2012).

The $\mathrm{pH}$ of culture medium increased during lipid production over time to values of 9 , due to VFAs consumption. At slightly acidic pH, VFA is mostly in undissociated form, which once inside the cell, dissociates intracellularly, and leads to cytosolic acidification, which may alter several metabolic pathways (Casal et al., 2008). In this turn, the adverse effects of VFAs at low concentration are weak and negligible (Fei et al., 2011b; Gao et al., 2017; Liu et al., 2017).

Despite of $Y$. lipolytica W29 readily consume VFAs, the addition of a co-substrate ( $5 \mathrm{~g} \cdot \mathrm{L}^{-1}$ glucose) to each VFA-based medium was tested as an attempt to improve yeast growth and, consequently, the titer of lipids produced. Approximately 2 -fold improvement on cellular concentration was observed with the addition of glucose, regardless of the VFA in the medium (Fig. 2A). Although no differences were observed on biomass yield (dry cell mass per mass of total substrates, VFAs and co-substrate, consumed) with the addition of glucose, the highest biomass production was attained with butyrate as occurred in experiments with single VFAs (Table 1).

In all experiments, cell proliferation started without lag phase and the stationary phase was attained after $24 \mathrm{~h}$ of yeast cultivation, similarly to the experiments with single VFA $(27 \mathrm{~h})$. The addition of a cosubstrate, easily assimilable by $Y$. lipolytica, changed the growth profile of the yeast. The specific growth rate on acetate $\left(0.185 \mathrm{~h}^{-1} \pm\right.$ $\left.0.002 \mathrm{~h}^{-1}\right)$, propionate $\left(0.147 \mathrm{~h}^{-1} \pm 0.003 \mathrm{~h}^{-1}\right)$ and butyrate $\left(0.200 \mathrm{~h}^{-1} \pm 0.001 \mathrm{~h}^{-1}\right)$ were $22 \%, 33 \%$ and $25 \%$, respectively, higher than those obtained in the absence of glucose. In the presence of glucose, all substrates (VFA + glucose) were consumed simultaneously with yeast growth, but the consumption of propionate was slower than other VFAs and glucose, and only after $48 \mathrm{~h}$ (stationary growth phase) was completely depleted from the culture medium (Fig. 2B). By contrast, acetate, butyrate and glucose were totally consumed after $24 \mathrm{~h}$ of cultivation, showing that $Y$. lipolytica W29 can effectively utilize VFAs and sugars simultaneously for cellular growth. In butyrate-based medium, the addition of glucose led to an increase of VFA uptake rate, whereas in propionate-based medium a decrease was observed with glucose supplementation (Table 1). In experiments with acetate, no differences were found with the addition of glucose. Furthermore, it is worth to notice that uptake rates of acetate and glucose were similar and 

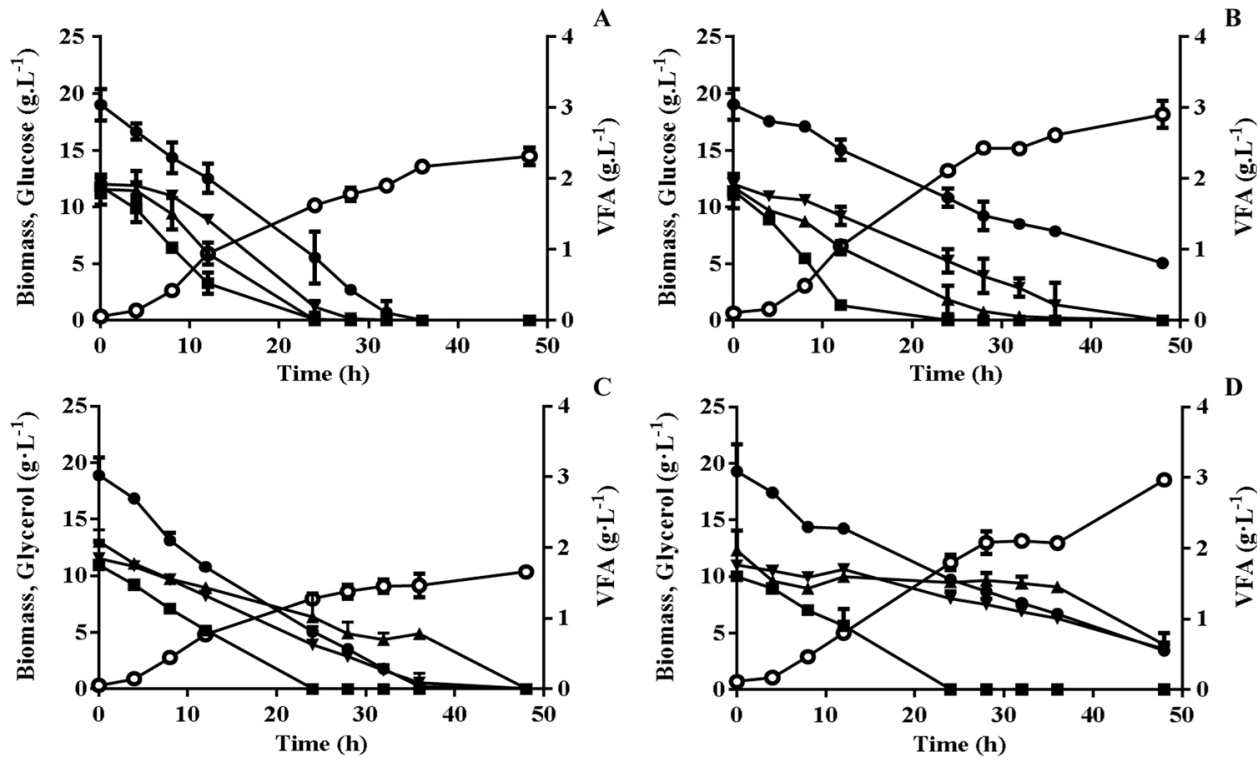

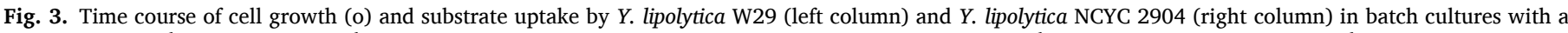

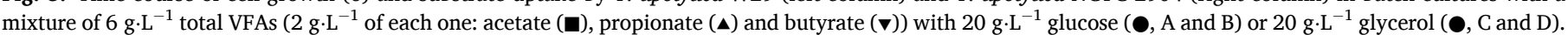
The error bars represent the standard deviation of two independent replicates.

higher than propionate and butyrate uptake rates (Table 1 ).

An improvement on lipid cellular content was observed by the addition of glucose as co-substrate, in cultures with acetate and propionate but was not affected for butyrate-based media. Around 1.2-fold enhancement on lipids production was obtained with the supplementation of glucose in acetate and propionate media. Glucose, under nitrogen limited condition, is metabolized by $Y$. lipolytica through glycolysis and tricarboxylic acid cycle to generate acetyl-CoA, which is then used for de novo fatty acid synthesis (Papanikolaou et al., 2009). Although the lipids content remained low (12.4\%), the increase of final biomass with the addition of glucose led to a 2- and 3-fold enhancement of microbial lipids concentration $\left(0.41 \mathrm{~g} \cdot \mathrm{L}^{-1}-0.85 \mathrm{~g} \cdot \mathrm{L}^{-1}\right)$ and consequently a 10 -fold improvement in lipid productivity comparatively with experiments with butyrate, propionate and acetate individually (Table 1). In previous works, the addition of glucose as co-substrate to acetate and propionate led to an enhancement of lipids content from $13.3 \%(\mathrm{w} / \mathrm{w})$ to $16.5 \%(\mathrm{w} / \mathrm{w})$ and from $8.9 \%(\mathrm{w} / \mathrm{w})$ to $10.2 \%(\mathrm{w} / \mathrm{w})$, respectively (Kolouchová et al., 2015). The lipids production by Y. lipolytica from frying vegetable oils enhanced from $25 \%(\mathrm{w} / \mathrm{w})$ to 55 $\%(\mathrm{w} / \mathrm{w})$ when medium was supplemented with glucose (Bialy et al., 2011).

Since cellular growth was enhanced by addition of glucose and, consequently, the amount of lipids synthesized also increased (Table 1), the raise of glucose concentration up to $20 \mathrm{~g} \cdot \mathrm{L}^{-1}$ was tested as an attempt to boost even more the concentration of biomass and microbial lipids. Additionally, the replacement of glucose by glycerol, a low-cost by-product of biodiesel industry, was tested. As the liquid broth of a real anaerobic digestion of organic wastes consists in a mixture of VFAs (Gao et al., 2017), batch cultures with a mixture of VFAs (with $2 \mathrm{~g} \cdot \mathrm{L}^{-1}$ acetate, $2 \mathrm{~g} \cdot \mathrm{L}^{-1}$ propionate and $2 \mathrm{~g} \cdot \mathrm{L}^{-1}$ butyrate) and $20 \mathrm{~g} \cdot \mathrm{L}^{-1}$ glucose or $20 \mathrm{~g} \cdot \mathrm{L}^{-1}$ glycerol were carried out. The mixture of VFAs was chosen as model based on total initial concentration of $6 \mathrm{~g} \cdot \mathrm{L}^{-1}$ of VFAs. Besides Y. lipolytica W29, also Y. lipolytica NCYC 2904 was studied in these experiments. This strain, isolated from a maize-processing plant (Illinois, USA), is less explored than W29 strain, though have already been demonstrated its potential to accumulate lipids from food processing by-products like okara (Vong et al., 2016).

In general, the simultaneous increase of glucose concentration and the use of a VFAs mixture did not affect the biomass yield. However, higher biomass concentration $\left(14.5 \mathrm{~g} \cdot \mathrm{L}^{-1} \pm 0.6 \mathrm{~g} \cdot \mathrm{L}^{-1}\right)$ was attained comparatively to previous experiments (Fig. 3A). The supplementation of $20 \mathrm{~g} \cdot \mathrm{L}^{-1}$ glucose essentially prevented $\mathrm{pH}$ changes when the yeast is growing on VFAs and thus improved the cellular growth. For $Y$. lipolytica growing on VFAs, the $\mathrm{pH}$ of culture medium increases to values above of 9, while in sugar media, the culture $\mathrm{pH}$ usually drops due to the production of organic acids (Papanikolaou et al., 2009). Yarrowia lipolytica NCYC 2904 was also able to grow in a mixture of VFAs supplemented with glucose with higher yield biomass $\left(0.93 \mathrm{~g} \cdot \mathrm{g}^{-1} \pm 0.05 \mathrm{~g} \cdot \mathrm{g}^{-1}\right)$, attaining high cell density comparatively to W29 strain cultures (Fig. 3A and 3B). In experiments with glycerol as co-substrate (Fig. 3C and 3D), the same behavior was observed and higher cellular concentration was reached in Y. lipolytica NCYC 2904 cultures. Moreover, the biomass yield was comparable to that obtained with glucose, whereas for W29 strain a significantly decrease was observed (Table 1 ). Glucose and VFAs were consumed simultaneously with yeasts growth, though W29 and NCYC 2904 strains had different consumption profiles. Whereas a complete depletion of glucose and VFAs were observed, respectively, after $36 \mathrm{~h}$ and $28 \mathrm{~h}$ for Y. lipolytica W29 (Fig. 3A), NCYC 2904 strain did not consume all glucose in the experiments time and the exhaustion of VFAs was observed only after $36 \mathrm{~h}$ (Fig. 3B). The global uptake rate of VFAs by Y. lipolytica NCYC 2904 was $24 \%$ lower than that attained by W29 strain. Similarly, glycerol was not totally consumed by NCYC 2904 strain (Fig. 3D), whereas in W29 strain cultures, this co-substrate was depleted from medium after $40 \mathrm{~h}$ (Fig. 3C). Moreover, the global uptake rate of VFAs were significantly lower in cultures with addition of glycerol than with glucose. Both yeasts showed a remarkable preference for acetate over propionate and butyrate, particularly the NCYC 2904 strain in cultures with glycerol. Furthermore, it is worth to notice that acetate was always consumed faster than other VFAs, regardless of experiments have been carried out with single VFAs or mixed with addition of glucose or glycerol. This is not surprising, since VFAs consumption is related with different metabolic pathways inside yeast cells, and this is not affected by the glucose or glycerol supplementation.

Although the low content of microbial lipids accumulated by Y. lipolytica W29 and NCYC 2904 strains of $10 \%(\mathrm{w} / \mathrm{w})$ and $13 \%(\mathrm{w} / \mathrm{w})$ respectively, an enhancement of lipids concentration as well as lipid productivity $\left(30 \mathrm{mg} \cdot \mathrm{L}^{-1} \cdot \mathrm{h}^{-1} \pm 6 \mathrm{mg} \cdot \mathrm{L}^{-1} \cdot \mathrm{h}^{-1}\right.$ ) were attained with the addition of glucose. For $Y$. lipolytica W29, a 3- and 2-fold improvement on intracellular lipids concentration was obtained, relatively to the experiments with butyrate as sole carbon source and butyrate 
Table 2

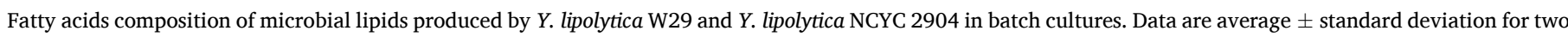
independent replicates.

\begin{tabular}{|c|c|c|c|c|c|c|c|}
\hline \multirow{2}{*}{ Y. lipolytica Strain } & \multirow{2}{*}{ Carbon Source } & \multicolumn{6}{|c|}{ Relative fatty acid content (\%) } \\
\hline & & C16:0 & C16:1 & C17:0 & C18:0 & C18:1 & C18:2 \\
\hline \multirow{8}{*}{ W29 } & Acetate & $3.8 \pm 0.3$ & $10 \pm 1$ & - & $1.0 \pm 0.8$ & $35.6 \pm 0.6$ & $49.6 \pm 0.9$ \\
\hline & Propionate & $2.9 \pm 0.6$ & $11.8 \pm 0.4$ & $6.1 \pm 0.5$ & $4.0 \pm 0.9$ & $34.4 \pm 0.9$ & $40.7 \pm 0.9$ \\
\hline & Butyrate & $8.3 \pm 0.3$ & $10.5 \pm 0.2$ & - & $4.8 \pm 0.7$ & $41.0 \pm 0.6$ & $36.7 \pm 0.4$ \\
\hline & Acetate + Glucose & $7.9 \pm 0.2$ & $11.0 \pm 0.3$ & - & $2.0 \pm 0.5$ & $38.0 \pm 0.6$ & $41 \pm 1$ \\
\hline & Propionate + Glucose & $13 \pm 2$ & $10.2 \pm 0.2$ & $4.9 \pm 0$ & $4.0 \pm 0.5$ & $35.4 \pm 0.4$ & $32 \pm 1$ \\
\hline & Butyrate + Glucose & $10.5 \pm 0.1$ & $9.9 \pm 0.3$ & - & $3.8 \pm 0.5$ & $43.0 \pm 0.9$ & $33 \pm 1$ \\
\hline & VFAs + Glucose & $17 \pm 1$ & $8.9 \pm 0.3$ & $2.9 \pm 0.4$ & $8 \pm 3$ & $44 \pm 4$ & $20 \pm 5$ \\
\hline & VFAs + Glycerol & $12.6 \pm 0.8$ & $4.8 \pm 0.2$ & $5.1 \pm 0.4$ & $6.5 \pm 0.3$ & $36 \pm 1$ & $35 \pm 1$ \\
\hline \multirow{2}{*}{ NCYC 2904} & VFAs + Glucose & $13.2 \pm 0.3$ & $9.9 \pm 0.3$ & $2.4 \pm 0.1$ & $4.7 \pm 0.3$ & $43 \pm 1$ & $27 \pm 2$ \\
\hline & VFAs + Glycerol & $11.9 \pm 0.5$ & $7.1 \pm 0.3$ & $2.4 \pm 0.2$ & $5.8 \pm 0.4$ & $45 \pm 1$ & $28 \pm 2$ \\
\hline
\end{tabular}

supplemented with glucose $5 \mathrm{~g} \cdot \mathrm{L}^{-1}$, respectively (Table 1 ). The amount of lipids produced by $Y$. lipolytica NCYC $2904\left(2.6 \mathrm{~g} \cdot \mathrm{L}^{-1} \pm 0.4 \mathrm{~g} \cdot \mathrm{L}^{-1}\right)$ was $45 \%$ higher than that obtained with W29 strain. Additionally, the lipid yield was 2-fold of that obtained with W29 strain, with an average lipid productivity of $54 \mathrm{mg} \cdot \mathrm{L}^{-1} \cdot \mathrm{h}^{-1} \pm 8 \mathrm{mg} \cdot \mathrm{L}^{-1} \cdot \mathrm{h}^{-1}$. The lipids yield $\left(0.144 \mathrm{~g} \cdot \mathrm{g}^{-1} \pm 0.005 \mathrm{~g} \cdot \mathrm{g}^{-1}\right)$ and lipids concentration $\left(2.69 \mathrm{~g} \cdot \mathrm{L}^{-1} \pm\right.$ $0.04 \mathrm{~g} \cdot \mathrm{L}^{-1}$ ) obtained for $Y$. lipolytica NCYC 2904 , using glycerol as cosubstrate, were 4.4- and 3.3-fold higher than those reached by W29 strain (Table 1), indicating the potential of this yeast NCYC 2904 strain to synthetize de novo lipids from VFAs. Though no significant differences on lipids production were observed between glucose and glycerol cultures of Y. lipolytica NCYC 2904, higher lipids productivity was obtained in glucose for W29 strain (Table 1).

Fatty acids composition of lipids produced from hydrophilic substrates is dependent on yeast specificity, substrates, as well as culture conditions. In this work, lipids produced by $Y$. lipolytica from VFAs are mainly composed by palmitic, palmitoleic, stearic, oleic and linoleic acids (Table 2). Regardless of yeast strain, VFAs type and concentration and presence of glucose or glycerol, lipids produced have a significant higher unsaturated fraction $(>73 \%, p<0.05)$ than saturated one, being composed of oleic (34 \% - $45 \%$ ) and linoleic acids (20 \% - $50 \%)$. However, the relative percentage of each fatty acid was dependent on substrates used. While using VFAs as sole carbon source $Y$. lipolytica W29 accumulated more oleic and linoleic acids, the addition of glucose or glycerol led to an enhancement of palmitic acid, a slight increase of oleic acid and a decrease of linoleic acid contents. Papanikolaou et al. (2009) reported that the increase of glucose concentration induced an increase of linoleic acid, but a decrease on linoleic acid (20\%) was observed in batch cultures with mixture of VFAs $\left(6 \mathrm{~g} \cdot \mathrm{L}^{-1}\right)$ and glucose $\left(20 \mathrm{~g} \cdot \mathrm{L}^{-1}\right)$. It is worth to notice that margaric acid is only synthetized when propionate is used as carbon source (individually or in a mixture with other VFAs). Since the metabolic pathways of lipids synthesis from all VFAs involve acetyl-CoA, fatty acids composition of microbial lipids from different VFAs are expected to be similar. However, propionate is metabolized via a different pathway, and this precursor (propionyl-CoA) synthetizes odd number fatty acids, such as margaric acid (Gao et al., 2017; Kolouchová et al., 2015). This result is utmost important, since odd chain fatty acids may improve the properties of biodiesel and be used as precursors for manufacturing agricultural chemicals (biocides), flavors and industrial chemicals (Park et al., 2018). Although the fatty acids profile of yeast strains tested herein indicated to depend on culture conditions, similar fatty acids compositions were reported to other Y. lipolytica strains using VFAs as carbon source (Gao et al., 2017, 2020). In other hydrophilic substrates, such as glycerol (Poli et al., 2014) and hydrophobic carbon sources, such as pork lard (Lopes et al., 2018) and waste cooking oils (Lopes et al., 2019), Y. lipolytica strains also accumulated preferentially oleic and linoleic acids, suggesting that lipogenic features of this yeast prevail over substrate nature. Other oleaginous yeasts, such as Cryptococcus albidus (Vajpeyi and Chandran, 2015) and Rhodosporidium toruloides (Huang et al., 2016), growing in VFAs-based media, produced lipids with a similar fatty acids composition of those obtained in this work.

\subsection{Two-stage batch cultures}

As was concluded above, the enhancement of cellular growth, by addition of glucose, lead to an increase of lipids production. Thus, a twostage batch culture was performed with a $24 \mathrm{~h}$ first stage of yeast growth on glucose before the addition of VFAs into the culture medium. Mixtures of acetate, propionate and butyrate with $2 \mathrm{~g} \cdot \mathrm{L}^{-1}, 6 \mathrm{~g} \cdot \mathrm{L}^{-1}, 8 \mathrm{~g} \cdot \mathrm{L}^{-1}$ and $10 \mathrm{~g} \cdot \mathrm{L}^{-1}$ of each one were added to the medium in the second stage. In general, VFAs derived from organic wastes ranged from $4 \mathrm{~g} \cdot \mathrm{L}^{-1}$ to 40 g.L $\mathrm{L}^{-1}$ (Gameiro et al., 2016; Gao et al., 2017, 2020; Llamas et al., 2020; Moretto et al., 2019).

In general, $Y$. lipolytica W29 growth and biomass yield diminished with the increase of VFAs concentration, with approximately a 2-fold decrease by raising the total amount of VFAs from $6 \mathrm{~g} \cdot \mathrm{L}^{-1}$ to $30 \mathrm{~g} \cdot \mathrm{L}^{-1}$ (Fig. 4A; Table 3). The adverse effects of VFAs on yeast cells are
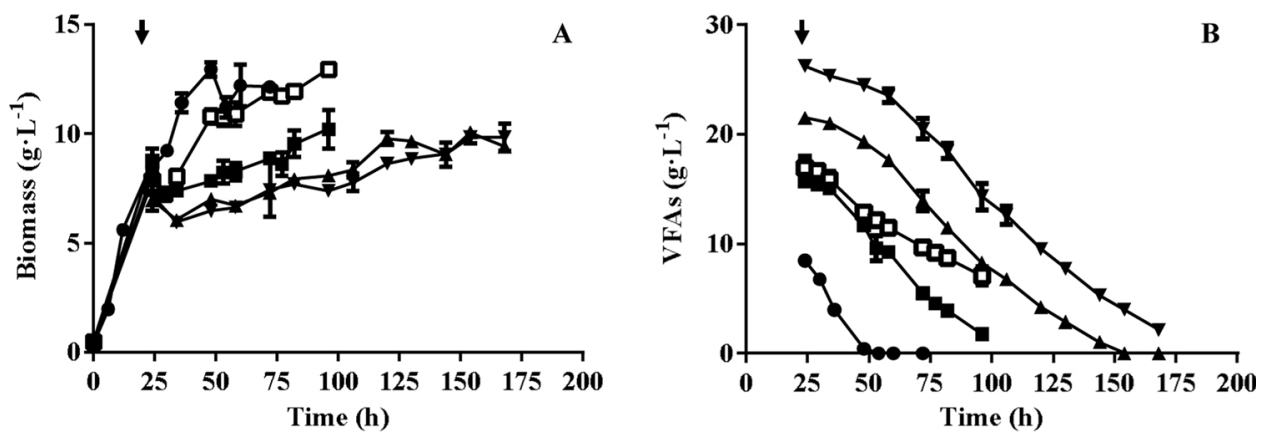

Fig. 4. Time course of cellular growth (A) and total VFAs uptake (B) in two-stage batch cultures of Y. lipolytica W29 (close symbols) and Y. lipolytica NCYC 2904 (open symbols). After $24 \mathrm{~h}$ of cellular growth in glucose $\left(20 \mathrm{~g} \cdot \mathrm{L}^{-1}\right)$, mixtures of $6 \mathrm{~g} \cdot \mathrm{L}^{-1}(\boldsymbol{\bullet}), 18 \mathrm{~g} \cdot \mathrm{L}^{-1}(\boldsymbol{\square}, \square), 24 \mathrm{~g} \cdot \mathrm{L}^{-1}(\boldsymbol{\Lambda})$ and $30 \mathrm{~g} \cdot \mathrm{L}^{-1}(\boldsymbol{\nabla})$ of total VFAs were added to culture medium. The error bars represent the standard deviation of two independent replicates. 
Table 3

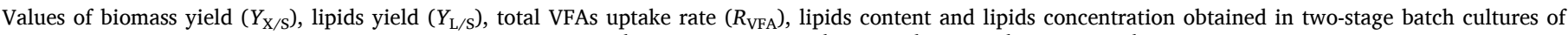

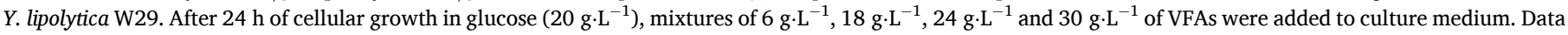

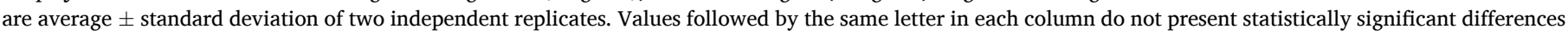
(95\% confidence level).

\begin{tabular}{|c|c|c|c|c|c|}
\hline VFAs Concentration & $Y_{\mathrm{X} / \mathrm{S}}\left(\mathrm{g} \cdot \mathrm{g}^{-1}\right)$ & $Y_{\mathrm{L} / \mathrm{S}}\left(\mathrm{g} \cdot \mathrm{g}^{-1}\right)$ & $R_{\mathrm{VFA}} *\left(\mathrm{~g} \cdot \mathrm{L}^{-1} \cdot \mathrm{h}^{-1}\right)$ & Lipids content $(\%, \mathrm{w} / \mathrm{w})$ & Lipids concentration $\left(\mathrm{g} \cdot \mathrm{L}^{-1}\right)$ \\
\hline $6 \mathrm{~g} \cdot \mathrm{L}^{-1}$ & $0.38 \pm 0.02^{\mathrm{a}}$ & $0.058 \pm 0.006^{\mathrm{a}}$ & $0.28 \pm 0.01^{\mathrm{a}}$ & $13.8 \pm 1.4^{\mathrm{a}}$ & $1.78 \pm 0.16^{\mathrm{a}}$ \\
\hline $18 \mathrm{~g} \cdot \mathrm{L}^{-1}$ & $0.26 \pm 0.02^{b}$ & $0.068 \pm 0.002^{\mathrm{a}}$ & $0.194 \pm 0.002^{b}$ & $25.4 \pm 0.9^{b}$ & $2.3 \pm 0.2^{b}$ \\
\hline $24 \mathrm{~g} \cdot \mathrm{L}^{-1}$ & $0.20 \pm 0.01^{c}$ & $0.042 \pm 0.004^{b}$ & $0.165 \pm 0.001^{b}$ & $17.5 \pm 1.6^{\mathrm{c}}$ & $1.28 \pm 0.09^{c}$ \\
\hline $30 \mathrm{~g} \cdot \mathrm{L}^{-1}$ & $0.20 \pm 0.01^{c}$ & $0.045 \pm 0.006^{b}$ & $0.167 \pm 0.000^{b}$ & $16.9 \pm 1.8^{\mathrm{c}}$ & $1.26 \pm 0.15^{c}$ \\
\hline
\end{tabular}

"Values correspond to second stage of cultivation.

commonly caused by undissociated form of VFA, which leads to intracellular acidification and induces stress on cell metabolism. Although yeasts possess a series of regulatory mechanisms to maintain a near neutral intracellular environment, high expenditure of ATP could interfere with membrane transport of phosphate and consequently affect yeast growth (Gao et al., 2017, 2020; Rodrigues and Pais, 2000). According to Fei et al. (2011b) and Fontanille et al. (2012), this inhibition was observed for an initial concentration of VFAs above $5 \mathrm{~g} \cdot \mathrm{L}^{-1}$. Moreover, different VFA types and initial concentrations could exert different intensities of inhibition. For instance, Gao et al. (2017) reported that high concentrations of VFAs inhibited cell growth in the following order: butyric acid $>$ propionic acid $>$ acetic acid. While $Y$. lipolytica CICC 31596 was able to grow on batch cultures with $20 \mathrm{~g} \cdot \mathrm{L}^{-1}$ acetic acid, no cellular growth was observed with $20 \mathrm{~g} \cdot \mathrm{L}^{-1}$ propionic acid and with concentrations above $10 \mathrm{~g} \cdot \mathrm{L}^{-1}$ of butyric acid. VFA ratio can also affects cellular growth, since the predominance of short-chain VFAs (e.g. acetic acid) in the culture medium has a positive effect on yeast growth, on the contrary to the presence of high proportion of long-chain VFAs (propionic and butyric acid) (Fei et al., 2011b; Llamas et al., 2020). Despite higher VFAs concentrations had affected adversely yeast growth, $Y$. lipolytica W29 was able to consume completely all VFAs, even in the experiments performed with $24 \mathrm{~g} \cdot \mathrm{L}^{-1}$ and $30 \mathrm{~g} \cdot \mathrm{L}^{-1}$ (Fig. 4B). Faster consumption and VFAs uptake rate were obtained with $6 \mathrm{~g} \cdot \mathrm{L}^{-1}$, but no significant differences were observed for results with VFAs concentration between $18 \mathrm{~g} \cdot \mathrm{L}^{-1}$ and $30 \mathrm{~g} \cdot \mathrm{L}^{-1}$ (Table 3). It is worth to notice that VFAs uptake rates attained in this work were considerable higher than others reported in literature. In Y. lipolytica CECT1240 cultures, $10.5 \mathrm{~g} \cdot \mathrm{L}^{-1}$ of VFAs mixture was totally consumed after $250 \mathrm{~h}$, while for $26.5 \mathrm{~g} \cdot \mathrm{L}^{-1} \mathrm{VFAs}$ mixture, only $7.6 \mathrm{~g} \cdot \mathrm{L}^{-1}$ was consumed after $380 \mathrm{~h}$ of culture (Llamas et al., 2020).

No improvement in biomass and lipids production was attained with the two-stage batch cultures of Y. lipolytica W29 using a mixture with total VFAs concentration of $6 \mathrm{~g} \cdot \mathrm{L}^{-1}$ (Table 3 ) relatively to batch culture of a mixture of VFAs $\left(6 \mathrm{~g} \cdot \mathrm{L}^{-1}\right)$ with glucose (Table 1). However, the global VFAs uptake rate of VFAs on two-stage batch culture $\left(0.28 \mathrm{~g} \cdot \mathrm{L}^{-1} \cdot \mathrm{h}^{-1}\right)$ was $33 \%$ higher $(p<0.05)$ than that obtained in batch culture $\left(0.21 \mathrm{~g} \cdot \mathrm{L}^{-1} \cdot \mathrm{h}^{-1}\right)$, due to the increased cellular concentration of the culture.

Though the highest biomass yield was obtained in cultures with lower amount of VFAs, the highest lipids yield, lipid content and lipid concentration were attained at total VFAs concentration of $18 \mathrm{~g} \cdot \mathrm{L}^{-1}$. In cultures with $24 \mathrm{~g} \cdot \mathrm{L}^{-1}$ and $30 \mathrm{~g} \cdot \mathrm{L}^{-1}$ of total VFAs, great amounts of VFA in unionized form are present, which may affect the activity of some enzymes involved in lipid synthesis (Gao et al., 2017). Gong et al. (2016)
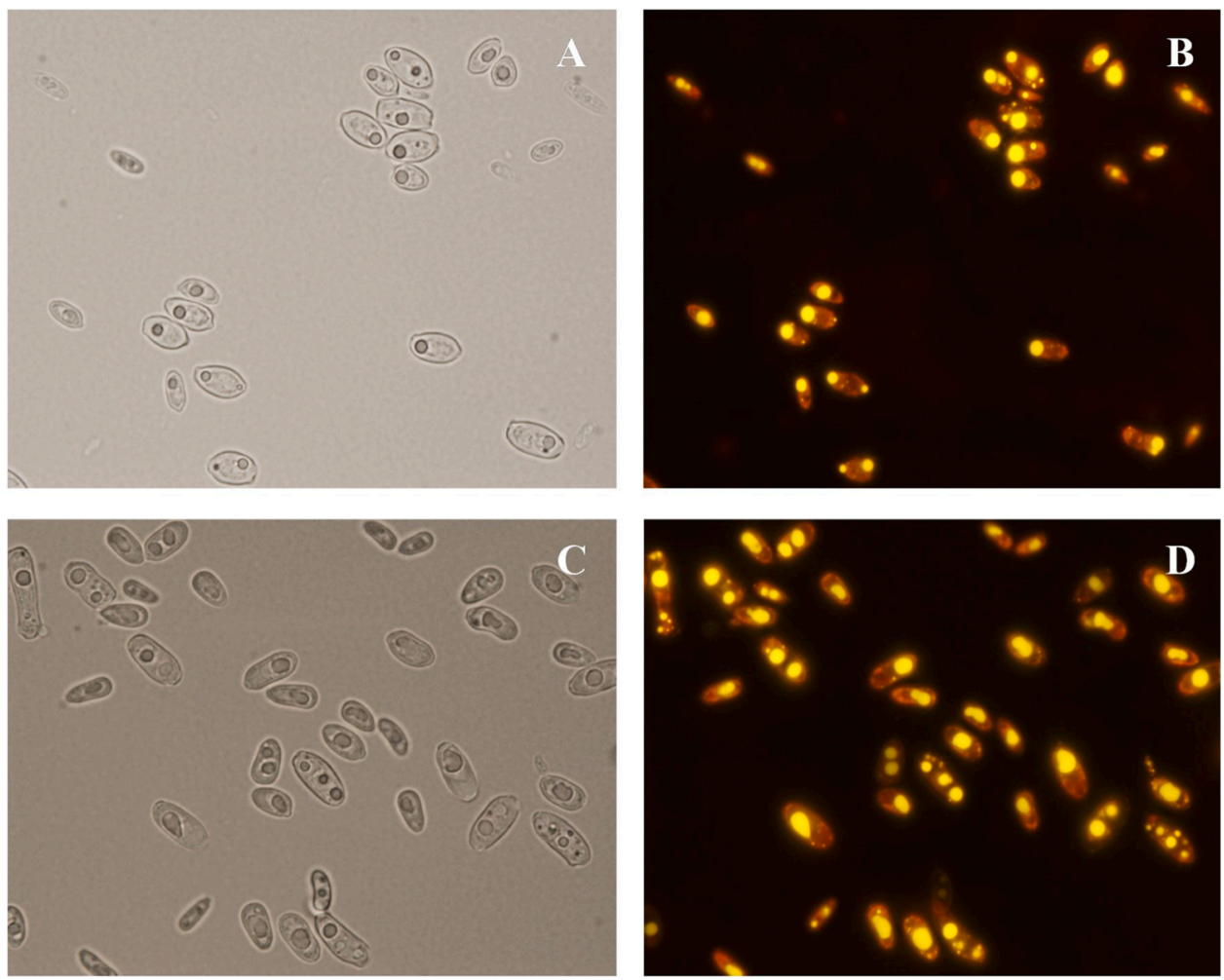

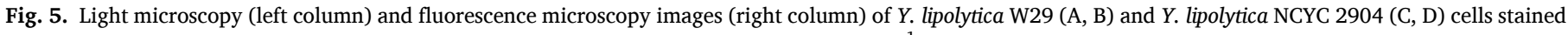
with Nile red: lipid bodies visualization after growth on two-stage batch culture with $18 \mathrm{~g} \cdot \mathrm{L}^{-1}$ VFAs (Magnification $1000 \mathrm{x}$ ). 
Table 4

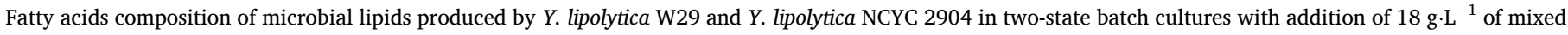
VFAs. Data are average \pm standard deviation for two independent replicates.

\begin{tabular}{|c|c|c|c|c|c|c|}
\hline \multirow{2}{*}{ Y. lipolytica Strain } & \multicolumn{6}{|c|}{ Relative fatty acid content (\%) } \\
\hline & $\mathrm{C} 16: 0$ & C16:1 & $\mathrm{C} 17: 0$ & C18:0 & C18:1 & C18:2 \\
\hline W29 & $11.7 \pm 0.6$ & $5.3 \pm 0.1$ & $3.0 \pm 0.1$ & $7.5 \pm 0.7$ & $51.0 \pm 0.5$ & $15.3 \pm 0.1$ \\
\hline NCYC 2904 & $9.6 \pm 0.2$ & $6.2 \pm 0.0$ & $2.2 \pm 0.2$ & $5.6 \pm 0.2$ & $53.8 \pm 0.2$ & $14.0 \pm 0.2$ \\
\hline
\end{tabular}

also observed a positive effect of increased acetate concentration up to $20 \mathrm{~g} \cdot \mathrm{L}^{-1}$ on lipids synthesis by C. curvatus ATCC 20509 , but above this amount the accumulation of lipids decreased considerably. Results obtained in two-stage batch culture with VFAs mixture of $30 \mathrm{~g} \cdot \mathrm{L}^{-1}$ were similar to attained in batch culture with $30 \mathrm{~g} \cdot \mathrm{L}^{-1}$ of acetate (Gao et al., 2020). Moreover, the authors showed that initial $\mathrm{pH}$ of culture medium had a crucial effect on yeast when high concentration of acetate was used and increasing $\mathrm{pH}$ to alkaline levels can alleviate the toxic effect of high content of VFAs. Thus, the slight acidic $\mathrm{pH}$ of culture medium in beginning of second stage (after addition of VFAs) could be contributed to inhibition of high VFAs concentration on lipids production.

As the two-stage batch cultures using a mixture of $18 \mathrm{~g} \cdot \mathrm{L}^{-1}$ VFAs proved to be effective for lipids synthesis enhancement by $Y$. lipolytica W29, and NCYC 2904 strain produced $41 \%$ higher lipids than W29 strain in batch cultures, this strategy was also tested with NCYC 2904 strain. The biomass yield $\left(0.46 \mathrm{~g} \cdot \mathrm{g}^{-1} \pm 0.07 \mathrm{~g} \cdot \mathrm{g}^{-1}\right)$ was higher than that for W29 strain, though total VFAs uptake rate $\left(0.138 \mathrm{~g} \cdot \mathrm{L}^{-1} \cdot \mathrm{h}^{-1} \pm\right.$
$0.002 \mathrm{~g} \cdot \mathrm{L}^{-1} \cdot \mathrm{h}^{-1}$ ) be considerably lower, and approximately $7 \mathrm{~g} \cdot \mathrm{L}^{-1}$ of VFAs remained in culture medium at the end of growth. In the beginning of second stage (addition of VFAs), $10 \mathrm{~g} \cdot \mathrm{L}^{-1}$ of glucose remained in Y. lipolytica NCYC 2904 cultivation medium, whereas in W29 cultures only $4 \mathrm{~g} \cdot \mathrm{L}^{-1}$ of glucose were not consumed. This may be the reason for the lower uptake rate of VFAs mixture obtained for NCYC 2904 strain. At the end of experiments, the lipids content $(26.7 \% \pm 1.6 \%, \mathrm{w} / \mathrm{w})$ and lipids concentration $\left(3.5 \mathrm{~g} \cdot \mathrm{L}^{-1} \pm 0.2 \mathrm{~g} \cdot \mathrm{L}^{-1}\right)$, were approximately 2 -fold higher than those attained by W29 strain, as occurred in batch cultures.

It is worth to notice that the amount of lipids produced in two-stage batch cultures of NCYC 2904 and W29 strains, with addition of $18 \mathrm{~g} \cdot \mathrm{L}^{-1}$ of VFAs mixture, led to a 2- and 2.5-fold improvement on lipids content comparatively to that obtained in batch cultures with a mixture of VFAs $\left(6 \mathrm{~g} \cdot \mathrm{L}^{-1}\right)$ and glucose $\left(20 \mathrm{~g} \cdot \mathrm{L}^{-1}\right)$. Although few authors have tested similar strategies for lipids production from VFAs by oleaginous yeasts in flask-scale, the results obtained herein are comparable or even higher than others found in the literature for C. curvatus, (Christophe et al.,
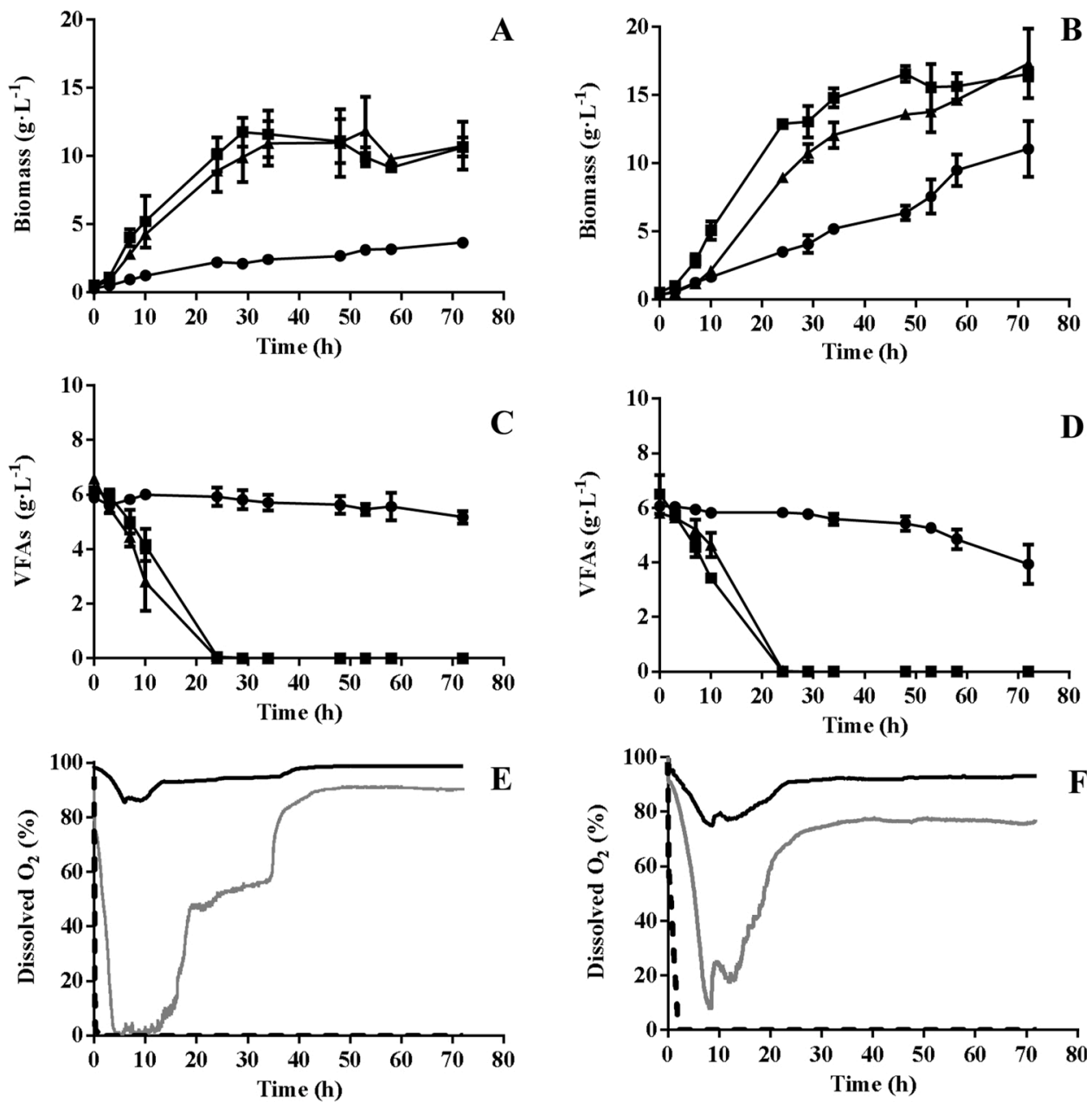

Fig. 6. Time course of cell growth (A, B), total VFAs uptake (C, D) and dissolved oxygen concentration (E, F) obtained in batch cultures of Y. lipolytica W29 (left column) and Y. lipolytica NCYC 2904 (right column) carried out in a STR bioreactor at different $k_{\mathrm{L}} a$ conditions: $22 \mathrm{~h}^{-1}$ (๑, black dashed line), $87 \mathrm{~h}^{-1}$ ( $\mathbf{\Lambda}$, grey line) and $125 \mathrm{~h}^{-1}$ (घ, black line). The error bars represent the standard deviation of two independent replicates. 
Table 5

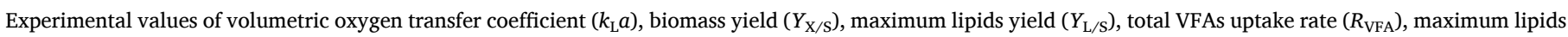

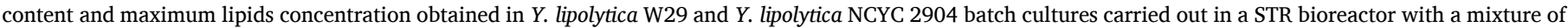

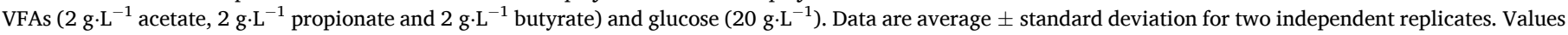
followed by the same letter in each column do not present statistically significant differences ( $95 \%$ confidence level).

\begin{tabular}{|c|c|c|c|c|c|c|}
\hline Strain & $k_{\mathrm{L}} a\left(\mathrm{~h}^{-1}\right)$ & $Y_{\mathrm{X} / \mathrm{s}}\left(\mathrm{g} \cdot \mathrm{g}^{-1}\right)$ & $Y_{\mathrm{L} / \mathrm{S}}{ }^{*}\left(\mathrm{~g} \cdot \mathrm{g}^{-1}\right)$ & $R_{\mathrm{VFA}}\left(\mathrm{g} \cdot \mathrm{L}^{-1} \cdot \mathrm{h}^{-1}\right)$ & Lipids content* $(\%, w / w)$ & Lipids concentration* $\left(g \cdot \mathrm{L}^{-1}\right)$ \\
\hline \multirow{3}{*}{ W29 } & 22 & $0.17 \pm 0.03^{\mathrm{a}}$ & $0.04 \pm 0.00^{\mathrm{a}}$ & $0.013 \pm 0.001^{\mathrm{a}}$ & $7.5 \pm 0.1^{\mathrm{a}}$ & $0.203 \pm 0.003^{\mathrm{a}}$ \\
\hline & 87 & $0.3 \pm 0.1^{\mathrm{ab}}$ & $0.054 \pm 0.006^{\mathrm{ab}}$ & $0.27 \pm 0.4^{b}$ & $11.5 \pm 0.5^{b}$ & $1.3 \pm 0.2^{\mathrm{b}}$ \\
\hline & 125 & $0.36 \pm 0.03^{a b c}$ & $0.034 \pm 0.006^{\mathrm{a}}$ & $0.28 \pm 0.01^{b}$ & $9.3 \pm 0.3^{c}$ & $1.0 \pm 0.2^{b}$ \\
\hline \multirow{3}{*}{ NCYC 2904} & 22 & $0.6 \pm 0.1^{c}$ & $0.09 \pm 0.02^{b c}$ & $0.058 \pm 0.001^{\mathrm{a}}$ & $12.2 \pm 0.8^{b d}$ & $1.3 \pm 0.2^{b}$ \\
\hline & 87 & $0.56 \pm 0.03^{b c}$ & $0.131 \pm 0.005^{d}$ & $0.264 \pm 0.006^{b}$ & $21.8 \pm 0.3^{f}$ & $3.60 \pm 0.09^{c}$ \\
\hline & 125 & $0.54 \pm 0.07^{b c}$ & $0.10 \pm 0.02^{c}$ & $0.242 \pm 0.005^{b}$ & $13 \pm 0.1^{\mathrm{d}}$ & $2.5 \pm 0.7^{\mathrm{d}}$ \\
\hline
\end{tabular}

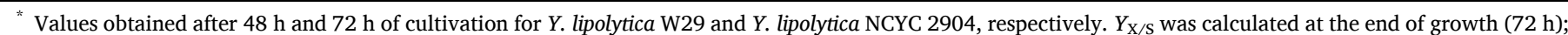
$R_{\mathrm{VFA}}$ was calculated in the first $24 \mathrm{~h}$ for experiments at $k_{\mathrm{L}} a$ of $87 \mathrm{~h}^{-1}$ and $125 \mathrm{~h}^{-1}$, and $72 \mathrm{~h}$ for $k_{\mathrm{L}} a$ of $22 \mathrm{~h}^{-1}$.

2012), C. albidus (Fei et al., 2011b) and R. toruloides (Huang et al., 2016).

The microscopic visualization of $Y$. lipolytica W29 (Fig. 5A) and Y. lipolytica NCYC 2904 (Fig. 5C) cells cultivated in medium with VFAs revealed that both yeast cells exhibited a typical oval form. Additionally, it was possible to clearly visualize the lipids accumulated in lipid bodies within the cells. The fluorescence microscopy after staining with Nile Red allows the clear visualization of lipids accumulated in lipid bodies, stained in yellow. Yarrowia lipolytica W29 cells (Fig. 5B) had one or two lipid bodies within the cells, while in Y. lipolytica NCYC 2904 cells (Fig. 5D) several lipid bodies inside the cells were observed, which is in accordance with higher lipid content reached by NCYC 2904 strain comparatively to W29 strain.

On whole, Erlenmeyer flask experiments demonstrated the ability of Y. lipolytica W29 and Y. lipolytica NCYC 2904 to accumulate lipids using VFAs as carbon source, and both strains showed high tolerance for assimilate VFAs at concentrations up to $18 \mathrm{~g} \cdot \mathrm{L}^{-1}$ in two-stage batch cultures. In this strategy, lipids produced by both strains are mainly composed by oleic acid ( $>50 \%$ ), followed by linoleic and stearic acids (Table 4). It should be noticed that the unsaturated fraction (72\% - 74 $\%$ ) largely exceeded the saturated one (17 \% - $22 \%)$, making these oils a plausible substitute of vegetable oils for biodiesel production.

\subsection{Effect of oxygen supply on microbial lipids production}

Dissolved oxygen concentration in cultivation medium is an operational parameter with great influence on $Y$. lipolytica growth and lipids production (Magdouli et al., 2018). Furthermore, oxygen mass transfer from gas phase to the liquid medium is a crucial variable for process scale-up, since $Y$. lipolytica is a strictly aerobic yeast. Therefore, the effect of $k_{\mathrm{L}} a$ on growth and lipids synthesis by $Y$. lipolytica from VFAs was studied. Both W29 and NCYC 2904 strains were studied in batch cultures in a STR lab-scale bioreactor using a medium with a mixture of $6 \mathrm{~g} \cdot \mathrm{L}^{-1}$ total VFAs and glucose $\left(20 \mathrm{~g} \cdot \mathrm{L}^{-1}\right)$. Different aeration and agitation rates were varied to obtain three different values of $k_{\mathrm{L}} a$ of $22 \mathrm{~h}^{-1} \pm 2 \mathrm{~h}^{-1}$ (at $0.5 \mathrm{vvm}$ and $200 \mathrm{rpm}$ ), $87 \mathrm{~h}^{-1} \pm 2 \mathrm{~h}^{-1}$ (at $1 \mathrm{vvm}$ and $400 \mathrm{rpm}$ ) and 125 $\mathrm{h}^{-1} \pm 1 \mathrm{~h}^{-1}$ (at $1.5 \mathrm{vvm}$ and $600 \mathrm{rpm}$ ). During the first hours of yeast cultivation (corresponding to the exponential growth phase), a decrease on oxygen concentration in the medium was observed, particularly in experiments carried out at $k_{\mathrm{L}} a$ of $22 \mathrm{~h}^{-1}$ and $87 \mathrm{~h}^{-1}$ (Fig. 6E and $6 \mathrm{~F}$ ). In the condition of lowest oxygen transfer rate, oxygen concentration dropped to zero in the first hour of growth, remaining in this value until the end of process. This can justify the low biomass production and VFAs consumption rate obtained in this condition, owing to an oxygen limitation (Fig. 6A and 6B). In the experiments conducted at $k_{\mathrm{L}} a$ of $87 \mathrm{~h}^{-1}$, the dissolved oxygen concentration dropped to zero (W29 strain) and to $8 \%$ (NCYC 2904 strain) in the first hours of cultivation, increased during stationary phase of growth and remained above $80 \%$ (W29) and 70\% (NCYC 2904) until the end of experiments. Contrariwise, for $k_{\mathrm{L}} a$ of $125 \mathrm{~h}^{-1}$, the dissolved oxygen concentration never fell to zero and stabilized at around $95 \%$ after exponential growth phase (Fig. $6 \mathrm{E}$ and $6 \mathrm{~F}$ ).
The increase of $K_{\mathrm{L}} a$ from $22 \mathrm{~h}^{-1}$ to $87 \mathrm{~h}^{-1}$ had a positive effect on cellular growth of Y. lipolytica W29 and NCYC 2904, but the further increase did not favor cellular growth and VFAs consumption (Table 5). As Y. lipolytica is a strictly aerobic yeast, the enhancement of cellular growth is expected by raising the oxygen mass transfer (Magdouli et al., 2018). Nevertheless, the mechanical stress promoted by high agitation rates or oxidative stress resulting from the high dissolved oxygen concentration may inhibit cellular metabolism (Alonso et al., 2005). Though no significant differences were observed on growth rate of $Y$. lipolytica NCYC $2904\left(0.29 \mathrm{~h}^{-1} \pm 0.07 \mathrm{~h}^{-1}\right)$ and W29 strain $\left(0.23 \mathrm{~h}^{-1} \pm 0.01 \mathrm{~h}^{-1}\right)$ at higher $k_{\mathrm{L}} a$ conditions, the final biomass of NCYC 2904 cultures was 40 $\%$ higher than that of W29 (Fig. 6A and 6B). As already observed in Erlenmeyer flask experiments, also in bioreactor experiments NCYC 2904 strain demonstrated an intrinsic ability to grow better than W29 strain on VFAs, regardless of oxygenation conditions. Notwithstanding the higher growth, total VFAs uptake rate of NCYC 2904 strain was similar to W29 strain (Table 5). The VFAs assimilation was clearly affected by oxygenation conditions and a $95 \%$ improvement was obtained by increasing $k_{\mathrm{L}} a$ from $22 \mathrm{~h}^{-1}$ to $87 \mathrm{~h}^{-1}$ or to $125 \mathrm{~h}^{-1}$. Whereas a complete consumption of VFAs was observed after $24 \mathrm{~h}$ for both strains at higher $k_{\mathrm{L}} a$ conditions, a significant amount of unconsumed VFAs remained in the culture medium in experiments carried out at $k_{\mathrm{L}} a$ of $22 \mathrm{~h}^{-1}$ (Fig. 6C and 6D). The VFAs uptake rate obtained at $k_{\mathrm{L}} a$ of $125 \mathrm{~h}^{-1}$ in batch cultures in bioreactor was $33 \%$ higher $(\mathrm{p}<0.05)$ than that obtained in Erlenmeyer flask experiments (VFAs + glucose, Table 1), showing the important role of oxygenation of culture medium in the VFAs metabolization by the cells.

The effect of dissolved oxygen on lipids accumulation by $Y$. lipolytica is not consensual and no information regarding its influence on lipids production by $Y$. lipolytica from VFAs is available. In this study, and regardless of yeast strain, a clearly positive effect was observed on lipids production by increasing $k_{\mathrm{L}} a$ to $87 \mathrm{~h}^{-1}$. Approximately 7 - and 3 -fold improvement on microbial lipids concentration was attained for Y. lipolytica W29 and NCYC 2904 cultures, respectively (Table 5). According to Bellou et al. (2014), lipid synthesis is upregulated under high dissolved oxygen concentrations as result of the upregulation of enzymes involved in lipids synthesis (ATP-citrate lyase and malic enzyme). However, the further increase of oxygenation led to a considerable reduction on lipids synthesis by Y. lipolytica NCYC 2904. It was already reported that, in some cases, high oxygen concentration during lipids biosynthesis drives the yeast metabolism toward the production of lipid-free biomass, instead of lipids synthesis (Papanikolaou et al., 2007). It is worth to notice that there was no improvement in lipids production by $Y$. lipolytica W29 in bioreactor experiments comparatively with flask experiments, while lipids synthesis by Y. lipolytica NCYC 2904 were $40 \%$ higher in this system. Unlike to Y. lipolytica NCYC 2904, that accumulated more intracellular lipids in batch cultures in a STR bioreactor, batch cultures may be not the most effective for lipids accumulation by Y. lipolytica W29. Among the experimental conditions studied, the highest lipids productivity was obtained in the experiments in which dissolved oxygen concentration remained above $70 \%$ in stationary 
Table 6

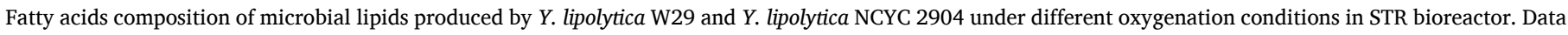
are average \pm standard deviation for two independent replicates.

\begin{tabular}{|c|c|c|c|c|c|c|c|}
\hline \multirow{2}{*}{ Strain } & \multirow{2}{*}{$k_{\mathrm{L}} a\left(\mathrm{~h}^{-1}\right)$} & \multicolumn{6}{|c|}{ Relative fatty acid content (\%) } \\
\hline & & $\mathrm{C} 16: 0$ & C16:1 & $\mathrm{C} 17: 0$ & C18:0 & C18:1 & C18:2 \\
\hline \multirow{3}{*}{ W29 } & 22 & $30 \pm 1$ & $3.1 \pm 0.2$ & - & $21.8 \pm 0.8$ & $21.0 \pm 0.9$ & $25.8 \pm 0.0$ \\
\hline & 87 & $15 \pm 1$ & $7.6 \pm 0.9$ & $9.2 \pm 0.6$ & $8 \pm 2$ & $38 \pm 2$ & $23 \pm 2$ \\
\hline & 125 & $16 \pm 2$ & $8.7 \pm 0.4$ & $5 \pm 1$ & $6.3 \pm 0.9$ & $44.0 \pm 0.8$ & $19.6 \pm 1.6$ \\
\hline \multirow{3}{*}{ NCYC 2904} & 22 & $11.4 \pm 0.5$ & $7.7 \pm 0.4$ & - & $8.6 \pm 0.3$ & $56.3 \pm 0.1$ & $16.7 \pm 0.6$ \\
\hline & 87 & $12.5 \pm 0.5$ & $12.4 \pm 0.1$ & $5.2 \pm 0.2$ & $4.9 \pm 0.6$ & $52 \pm 1$ & $13 \pm 1$ \\
\hline & 125 & $12.2 \pm 0.4$ & $11.2 \pm 0.5$ & $4.7 \pm 0.3$ & $4.9 \pm 0.6$ & $50 \pm 1$ & $16 \pm 1$ \\
\hline
\end{tabular}

growth phase $\left(k_{\mathrm{L}} a\right.$ of $\left.87 \mathrm{~h}^{-1}\right)$, that corresponds to the lipogenic phase in hydrophilic substrates. The increase of lipids synthesis by $Y$. lipolytica strains using highly aerated cultures was reported for glucose (Bellou et al., 2014; Tai and Stephanopoulos, 2013) and crude glycerol (Rakicka et al., 2015). By contrast, some authors demonstrated that lipids production by Y. lipolytica from crude glycerol (Magdouli et al., 2018), waste cooking oils (Lopes et al., 2019) and pork lard (Lopes et al., 2018) was higher under low dissolved oxygen concentrations. Other microbial species, such as Rhodotorula glutinis growing in glucose (Yen and Zhang, 2011) and C. albidus growing in VFAs (Fei et al., 2011a) enhanced their intracellular lipids content in conditions of poor oxygenation.

The results obtained during this study show that $k_{\mathrm{L}} a$ is an important factor on microbial lipids production by $Y$. lipolytica strains from VFAs and higher lipids synthesis from VFAs is reached at $k_{\mathrm{L}} a$ of $87 \mathrm{~h}^{-1}$.

Lipids accumulated by $Y$. lipolytica strains in bioreactor was mainly composed by oleic acid ( $21 \%$ - $56 \%$ ), followed by linoleic (13\% - $26 \%$ ) and palmitic (11\% - $30 \%$ ) acids (Table 6). Interestingly, at low $k_{\mathrm{L}} a$ condition, $Y$. lipolytica W29 accumulated more saturated fatty acids than unsaturated one, which is not very common in this yeast. Furthermore, at low oxygenation conditions, margaric acid was not synthetized by both strains since propionate was not assimilated. It seems that there is a relation between oxygenation conditions and fatty acids composition, which may be interest to produce tailor-made lipids with specific fatty acids composition. For $Y$. lipolytica W29, the unsaturated fraction increased with $k_{\mathrm{L}} a$ values, attaining the highest unsaturated fraction (> $70 \%$ ) at $k_{\mathrm{L}} a$ of $100 \mathrm{~h}^{-1}$. Lopes et al. (2018) also concluded that the unsaturated fraction of microbial lipids accumulated by $Y$. lipolytica W29 from pork lard was high in highly aerated cultures. Although the unsaturated fraction of microbial lipids synthesized by NCYC 2904 decreased with $k_{\mathrm{L}} a$ increase, its composition exceeds $77 \%$. Notwithstanding the differences in fatty acid profiles of lipids accumulated by the two Y. lipolytica strains, composition of fatty acids are similar to vegetable oils (rapeseed oil), with higher content in oleic and linoleic acids (Issariyakul and Dalai, 2014), suggesting that microbial lipids produced by Y. lipolytica W29 and Y. lipolytica NCYC 2904 from VFAs have great potential to be used for biodiesel production.

\section{Conclusions}

In this study, it was shown the ability of $Y$. lipolytica strains to grow on VFAs (individually or mixed with addition of glucose or glycerol) and accumulate lipids intracellularly. The highest lipids concentrations reached in Y. lipolytica W29 $\left(1.42 \mathrm{~g} \cdot \mathrm{L}^{-1}\right)$ and NCYC 2904 strain $\left(2.6 \mathrm{~g} \cdot \mathrm{L}^{-1}\right)$ batch cultures were obtained in experiments carried out with a mixture of VFAs supplemented with glucose $\left(20 \mathrm{~g} \cdot \mathrm{L}^{-1}\right)$. Moreover, the addition of co-substrates, like glucose or glycerol, suggest that the feasibility of this process may be improved by using simple sugars derived from agro-food wastes hydrolysis and food processing byproducts (e.g. molasses), or with others industrial by-products such as crude glycerol from biodiesel production. In two-stage batch experiments, with the addition of $18 \mathrm{~g} \cdot \mathrm{L}^{-1}$ of mixed VFAs after yeast growth on glucose, Y. lipolytica NCYC 2904 accumulated $34 \%$ more intracellular lipids than W29 strain. In lab-scale stirred bioreactor, culture oxygenation is an important factor affecting VFAs uptake rate and microbial lipids production. Whereas the increase of $k_{\mathrm{L}} a$ had no significant impact on yeast growth, the total VFAs uptake rate was maximum at $k_{\mathrm{L}} a$ of $87 \mathrm{~h}^{-1}$. The highest lipids synthesis was also reached in experiments conducted at $k_{\mathrm{L}} a$ of $87 \mathrm{~h}^{-1}$. The fatty acids composition similar to vegetable oils - higher content in oleic and linoleic acids - found in lipids accumulated by both $Y$. lipolytica strains, demonstrated its great potential to be used as feedstock in biodiesel industry. The results presented herein provide valuable information for using VFAs as substrate for lipids production by oleaginous yeasts. The bioconversion of VFAs, which are generated during acidogenesis of organic municipal solid wastes, by $Y$. lipolytica can be an important step in the valorization of these residues. The exploitation of oleaginous yeasts as biorefinery platform to obtain valuable compounds (microbial lipids) from low-cost substrates (VFAs produced from biodegradable municipal wastes) is an interesting strategy inserted in the circular economy concept.

\section{CRediT authorship contribution statement}

Ana S. Pereira: Methodology, Validation, Formal analysis, Investigation, Writing - original draft, Writing - review \& editing, Visualization. Sílvia M. Miranda: Methodology, Validation, Formal analysis, Investigation, Visualization. Marlene Lopes: Conceptualization, Methodology, Validation, Writing - original draft, Writing - review \& editing, Visualization, Supervision. Isabel Belo: Conceptualization, Methodology, Validation, Writing - original draft, Writing - review \& editing, Visualization, Supervision.

\section{Declaration of Competing Interest}

The authors report no declarations of interest.

\section{Acknowledgements}

This study was supported by the Portuguese Foundation for Science and Technology (FCT) under the scope of the project TUBITAK 2014 (TUBITAK/0009/2014), the strategic funding of UIDB/04469/2020 unit and Doctoral grants (SFRH/BD/144188/2019; SFRH/BD/129592/ 2017).

\section{References}

Alonso, F.O.M., Oliveira, E.B.L., Dellamora-Ortiz, G.M., Pereira-Meirelles, F.V., 2005. Improvement of lipase production at different stirring speeds and oxygen levels. Braz. J. Chem. Eng. 22, 9-18. https://doi.org/10.1590/S010466322005000100002.

Bellou, S., Makri, A., Triantaphyllidou, I.-E., Papanikolaou, S., Aggelis, G., 2014. Morphological and metabolic shifts of Yarrowia lipolytica induced by alteration of the dissolved oxygen concentration in the growth environment. Microbiology 160, 807-817. https://doi.org/10.1099/mic.0.074302-0.

Bialy, H..El, Gomaa, O.M., Azab, K.S., 2011. Conversion of oil waste to valuable fatty acids using Oleaginous yeast. World J. Microbiol. Biotechnol. 27, 2791-2798. https://doi.org/10.1007/s11274-011-0755-x.

Braga, A., Mesquita, D.P., Amaral, A.L., Ferreira, E.C., Belo, I., 2015. Aroma production by Yarrowia lipolytica in airlift and stirred tank bioreactors: differences in yeast metabolism and morphology. Biochem. Eng. J. 93, 55-62. https://doi.org/10.1016/ j.bej.2014.09.006. 
Casal, M., Paiva, S., Queirós, O., Soares-Silva, I., 2008. Transport of carboxylic acids in yeasts. FEMS Microbiol. Rev. 32, 974-994. https://doi.org/10.1111/j.1574. 6976.2008.00128.x.

Christophe, G., Deo, J.L., Kumar, V., Nouaille, R., Fontanille, P., Larroche, C., 2012. Production of oils from acetic acid by the oleaginous yeast Cryptococcus curvatus. Appl. Biochem. Biotechnol. 167, 1270-1279. https://doi.org/10.1007/s12010-011 9507-5.

Fei, Q., Chang, H.N., Shang, L., Choi, J., 2011a. Exploring low-cost carbon sources for microbial lipids production by fed-batch cultivation of Cryptococcus albidus. Biotechnol. Bioprocess Eng. 16, 482-487. https://doi.org/10.1007/s12257-0100370-y.

Fei, Q., Nam, H., Shang, L., Choi, J., Kim, N., Kang, J., 2011b. The effect of volatile fatty acids as a sole carbon source on lipid accumulation by Cryptococcus albidus for biodiesel production. Bioresour. Technol. 102, 2695-2701. https://doi.org/ 10.1016/j.biortech.2010.10.141.

Ferreira, P., Lopes, M., Mota, M., Belo, I., 2016. Oxygen mass transfer impact on citric acid production by Yarrowia lipolytica from crude glycerol. Biochem. Eng. J. 110, 35-42. https://doi.org/10.1016/j.bej.2016.02.001.

Fontanille, P., Kumar, V., Christophe, G., Nouaille, R., Larroche, C., 2012. Bioconversion of volatile fatty acids into lipids by the oleaginous yeast Yarrowia lipolytica. Bioresour. Technol. 114, 443-449. https://doi.org/10.1016/j.biortech.2012.02.091.

Gaeta-Bernardi, A., Parente, V., 2016. Organic municipal solid waste (MSW) as feedstock for biodiesel production: a financial feasibility analysis. Renew. Energy 86, 1422-1432. https://doi.org/10.1016/j.renene.2015.08.025.

Gameiro, T., Lopes, M., Marinho, R., 2016. Hydrolytic-Acidogenic Fermentation of Organic Solid Waste for Volatile Fatty Acids Production at Different Solids Concentrations and Alkalinity Addition. Water Air Soil Pollut. 227, 1-16. https:// doi.org/10.1007/s11270-016-3086-6.

Gao, R., Li, Z., Zhou, X., Cheng, S., Zheng, L., 2017. Oleaginous yeast Yarrowia lipolytica culture with synthetic and food waste - derived volatile fatty acids for lipid production. Biotechnol. Biofuels 10, 247. https://doi.org/10.1186/s13068-0170942-6.

Gao, R., Li, Z., Zhou, X., Bao, W., Cheng, S., Zheng, L., 2020. Enhanced lipid production by Yarrowia lipolytica cultured with synthetic and waste-derived high-content volatile fatty acids under alkaline conditions. Biotechnol. Biofuels 13, 3. https://doi. org/10.1186/s13068-019-1645-y.

Gong, Z., Zhou, W., Shen, H., Yang, Z., Wang, G., Zuo, Z., Hou, Y., Zhao, Z.K., 2016. Cofermentation of acetate and sugars facilitating microbial lipid production on acetaterich biomass hydrolysates. Bioresour. Technol. 207, 102-108. https://doi.org/ 10.1016/j.biortech.2016.01.122.

Huang, X.-F., Liu, J.-N., Lu, L.-J., Peng, K.-M., Yang, G.-X., Liu, J., 2016. Culture strategies for lipid production using acetic acid as sole carbon source by Rhodosporidium toruloides. Bioresour. Technol. 206, 141-149. https://doi.org/ 10.1016/j.biortech.2016.01.073.

Issariyakul, T., Dalai, A.K., 2014. Biodiesel from vegetable oils. Renewable Sustainable Energy Rev. 31, 446-471. https://doi.org/10.1016/j.rser.2013.11.001.

Katre, G., Joshi, C., Khot, M., Zinjarde, S., Ravikumar, A., 2012. Evaluation of single cell oil (SCO) from a tropical marine yeast Yarrowia lipolytica NCIM 3589 as a potential feedstock for biodiesel. AMB Express 2, 36. https://doi.org/10.1186/2191-0855-236.

Kolouchová, I., Schreiberová, O., Sigler, K., Masak, J., Řezanka, T., 2015. Biotransformation of volatile fatty acids by oleaginous and non-oleaginous yeast species. FEMS Yeast Res. 15. https://doi.org/10.1093/femsyr/fov076.

Lee, W.S., Chua, A.S.M., Yeoh, H.K., Ngoh, G.C., 2014. A review of the production and applications of waste-derived volatile fatty acids. Chem. Eng. J. 235, 83-99. https:// doi.org/10.1016/j.cej.2013.09.002.

Liu, N., Qiao, K., Stephanopoulos, G., 2016. C Metabolic Flux Analysis of acetate conversion to lipids by Yarrowia lipolytica. Metab. Eng. 38, 86-97. https://doi.org/ 10.1016/j.ymben.2016.06.006.

Liu, J., Yuan, M., Liu, J.-N., Huang, X.-F., 2017. Bioconversion of mixed volatile fatty acids into microbial lipids by Cryptococcus curvatus ATCC 20509. Bioresour. Technol. 241, 645-651. https://doi.org/10.1016/j.biortech.2017.05.085.

Llamas, M., Tomás-Pejó, E., González-Fernández, C., 2020. Volatile fatty acids from organic wastes as novel low-cost carbon sourve for Yarrowia lipolytica. N. Biotechnol. 56, 123-129. https://doi.org/10.1016/j.nbt.2020.01.002.

Lopes, M., Gomes, N., Mota, M., Belo, I., 2009. Yarrowia lipolytica growth under increased air pressure: influence on enzyme production. Appl. Biochem. Biotechnol. 159, 46-53. https://doi.org/10.1007/s12010-008-8359-0.

Lopes, M., Gomes, A.S., Silva, C.M., Belo, I., 2018. Microbial lipids and added value metabolites production by Yarrowia lipolytica from pork lard. J. Biotechnol. 265, 76-85. https://doi.org/10.1016/j.jbiotec.2017.11.007.
Lopes, M., Miranda, S.M., Alves, J.M., Pereira, A.S., Belo, I., 2019. Waste cooking oils as feedstock for lipase and lipid-rich biomass production. Eur. J. Lipid Sci. Technol. 121, 1800188 https://doi.org/10.1002/ejlt.201800188.

Magdouli, S., Brar, S.K., Blais, J.F., 2018. Morphology and rheological behaviour of Yarrowia lipolytica: impact of dissolved oxygen level on cell growth and lipid composition. Process Biochem. 65, 1-10. https://doi.org/10.1016/j. procbio.2017.10.021.

Moretto, G., Valentino, F., Pavan, P., Majone, M., Bolzonella, D., 2019. Optimization of urban waste fermentation for volatile fatty acids production. Waste Manag. 92, 21-29. https://doi.org/10.1016/j.wasman.2019.05.010.

Papanikolaou, S., Chevalot, I., Galiotou-panayotou, M., Komaitis, M., Marc, I., Aggelis, G., 2007. Industrial derivative of tallow: a promising renewable substrate for microbial lipid, single-cell protein and lipase production by Yarrowia lipolytica. Electron. J. Biotechnol. 10, 425-435. https://doi.org/10.2225/vol10-issue3-fulltext8.

Papanikolaou, S., Chatzifragkou, A., Fakas, S., Galiotou-Panayotou, M., Komaitis, M., Nicaud, J.-M., Aggelis, G., 2009. Biosynthesis of lipids and organic acids by Yarrowia lipolytica strains cultivated on glucose. Eur. J. Lipid Sci. Technol. 111, 1221-1232. https://doi.org/10.1002/ejlt.200900055.

Park, G.W., Chang, H.N., Jung, K., Seo, C., Kim, Y.-C., Choi, J.H., Woo, H.C., Hwang, I., 2017. Production of microbial lipid by Cryptococcus curvatus on rice straw hydrolysates. Process Biochem. 56, 147-153. https://doi.org/10.1016/j. procbio.2017.02.020.

Park, Y.K., Dulermo, T., Amaro, R.L., Nicaud, J.M., 2018. Biotechnology for Biofuels Optimization of odd chain fatty acid production by Yarrowia lipolytica. Biotechnol. Biofuels 11, 1-12. https://doi.org/10.1186/s13068-018-1154-4.

Poli, J.S., da Silva, M.A.N., Siqueira, E.P., Pasa, V.M.D., Rosa, C.A., Valente, P., 2014 Microbial lipid produced by Yarrowia lipolytica QU21 using industrial waste: a potential feedstock for biodiesel production. Bioresour. Technol. 161, 320-326. https://doi.org/10.1016/j.biortech.2014.03.083.

PORTDATA, 2020a. Municipal Waste Generation [WWW Document]. URL https://www. pordata.pt/en/Europe/Municipal+waste+generation-3423 (accessed 10.14.20).

PORTDATA, 2020b. Recycling Rate of Municipal Waste (\%) [WWW Document]. URL https://www.pordata.pt/en/Europe/Recycling + rate + of + municipal + waste + (percentage)-3426 (accessed 10.14.20).

Rakicka, M., Lazar, Z., Dulermo, T., Fickers, P., Nicaud, J.M., 2015. Lipid production by the oleaginous yeast Yarrowia lipolytica using industrial by - products under different culture conditions. Biotechnol. Biofuels 8, 1-10. https://doi.org/10.1186/s13068015-0286-Z.

Rodrigues, G., Pais, C., 2000. The influence of acetic and other weak carboxylic acids on growth and cellular death of the yeast yarrowia lipolytica the influence of acetic and other weak carboxylic acids on growth and cellular death of the yeast yarrowia. Food Technol. Biotechnol. 38, 27-32.

Sara, M., Brar, S.K., Blais, J.F., 2016. Lipid production by Yarrowia lipolytica grown on biodiesel-derived crude glycerol: optimization of growth parameters and their effects on the fermentation efficiency. RSC Adv. 6, 90547-90558. https://doi.org/10.1039/ C6RA16382C.

Scherhaufer, S., Moates, G., Hartikainen, H., Waldron, K., Obersteiner, G., 2018. Environmental impacts of food waste in Europe. Waste Manag. 77, 98-113. https:// doi.org/10.1016/j.wasman.2018.04.038.

Strazzera, G., Battista, F., Garcia, N.H., Frison, N., Bolzonella, D., 2018. Volatile fatty acids production from food wastes for biorefinery platforms: a review. J. Environ. Manage. 226, 278-288. https://doi.org/10.1016/j.jenvman.2018.08.039.

Tai, M., Stephanopoulos, G., 2013. Engineering the push and pull of lipid biosynthesis in oleaginous yeast Yarrowia lipolytica for biofuel production. Metab. Eng. 15, 1-9. https://doi.org/10.1016/j.ymben.2012.08.007.

Tsigie, Y.A., Wang, C., Kasim, N.S., Diem, Q., Huynh, L., Ho, Q., Truong, C., Ju, Y., 2012. Oil Production from Yarrowia lipolytica Po1g using rice bran hydrolysate. J. Biomed. Biotechnol. 2012. https://doi.org/10.1155/2012/378384.

Vajpeyi, S., Chandran, K., 2015. Microbial conversion of synthetic and food wastederived volatile fatty acids to lipids. Bioresour. Technol. 188, 49-55. https://doi. org/10.1016/j.biortech.2015.01.099.

Vong, W.C., Au Yang, K.L.C., Liu, S.-Q., 2016. Okara (soybean residue) biotransformation by yeast Yarrowia lipolytica. Int. J. Food Microbiol. 235, 1-9. https://doi.org/ 10.1016/j.ijfoodmicro.2016.06.039.

Yen, H.-W., Zhang, Z., 2011. Effects of dissolved oxygen level on cell growth and total lipid accumulation in the cultivation of Rhodotorula glutinis. J. Biosci. Bioeng. 112, 71-74. https://doi.org/10.1016/j.jbiosc.2011.03.013.

Zheng, Y., Chi, Z., Ahring, B.K., Chen, S., 2012. Oleaginous yeast Cryptococcus curvatus for biofuel production: ammonia's effect. Biomass Bioenergy 37, 114-121. https:// doi.org/10.1016/j.biombioe.2011.12.022. 